\section{Identification and Roles of Photosystem II Assembly, Stability, and Repair Factors in Arabidopsis}

\author{
Yan Lu* \\ Department of Biological Sciences, Western Michigan University, Kalamazoo, MI, USA
}

Photosystem II (PSII) is a multi-component pigment-protein complex that is responsible for water splitting, oxygen evolution, and plastoquinone reduction. Components of PSII can be classified into core proteins, low-molecular-mass proteins, extrinsic oxygen-evolving complex (OEC) proteins, and light-harvesting complex II proteins. In addition to these PSII subunits, more than 60 auxiliary proteins, enzymes, or components of thylakoid protein trafficking/targeting systems have been discovered to be directly or indirectly involved in de novo assembly and/or the repair and reassembly cycle of PSII. For example, components of thylakoid-protein-targeting complexes and the chloroplast-vesicle-transport system were found to deliver PSII subunits to thylakoid membranes. Various auxiliary proteins, such as PsbP-like (Psb stands for PSII) and light-harvesting complex-like proteins, atypical short-chain dehydrogenase/reductase family proteins, and tetratricopeptide repeat proteins, were discovered to assist the de novo assembly and stability of PSII and the repair and reassembly cycle of PSII. Furthermore, a series of enzymes were discovered to catalyze important enzymatic steps, such as C-terminal processing of the D1 protein, thiol/disulfide-modulation, peptidylprolyl isomerization, phosphorylation and dephosphorylation of PSII core and antenna proteins, and degradation of photodamaged PSII proteins. This review focuses on the current knowledge of the identities and molecular functions of different types of proteins that influence the assembly, stability, and repair of PSII in the higher plant Arabidopsis thaliana.

Keywords: Photosystem II assembly, Photosystem II stability, Photosystem II repair, Arabidopsis thaliana, identification and roles

\section{INTRODUCTION}

Photosystem II (PSII) is a multi-subunit pigment-protein complex found in thylakoid membranes of oxygenic photosynthetic organisms, including cyanobacteria, algae, and plants (Nickelsen and Rengstl, 2013; Järvi et al., 2015). Driven by light, PSII catalyzes electron transfer from water to plastoquinone. Therefore, PSII is also known as a water-plastoquinone oxidoreductase. Proteomics, X-ray crystallography, and single-particle electron cryo-microscopy studies revealed that PSII components include core proteins, low-molecular-mass (LMM, i.e., $<10 \mathrm{kDa}$ ) proteins, extrinsic oxygen-evolving complex (OEC) proteins, and light-harvesting complex (LHC) proteins (da Fonseca et al., 2002; Kashino et al., 2002; Liu et al., 2004; Aro et al., 2005; Nield and Barber, 2006; Umena et al., 2011; Suga et al., 2015). Except for some minor differences in the composition of LMM proteins, the core of PSII is conserved from cyanobacteria to land plants 
(Umena et al., 2011; Nickelsen and Rengstl, 2013). Proteins that form the PSII core complex in land plants include PSII reaction center core proteins D1 and D2 (i.e., PsbA and PsbD; Psb stands for PSII), core antenna proteins CP43 and CP47 (i.e., PSII chlorophyll proteins of 43 and $47 \mathrm{kDa}$, also known as PsbC and $\mathrm{PsbB}$, respectively), cytochrome $b_{559}$ subunits alpha and beta (i.e., PsbE and PsbF), and LMM proteins PsbH, PsbI, PsbJ, PsbK,

Abbreviations: ALB3, Albino3; BN-PAGE, blue native-polyacrylamide ge electrophoresis; CP43, Photosystem II chlorophyll protein of $43 \mathrm{kDa}$, also known as PsbC; CP47, Photosystem II chlorophyll protein of $47 \mathrm{kDa}$, also known as PsbB; CPRabA5e, chloroplast Rab GTPase A5e; cpFtsY, filamentation temperature sensitive protein $\mathrm{Y}$; $\mathrm{cpSec}$, chloroplast secretory; $\mathrm{cpSecA}, \mathrm{cpSecE}$, cpSecY, chloroplast secretory translocase A, E, and Y; cpSRP, chloroplast signal recognition particle; cpSRP43 and cpSRP54, chloroplast Signal Recognition Particle protein of 43 and $54 \mathrm{kDa}$; cpTat, chloroplast twin-arginine translocation; cpTatC, chloroplast twin-arginine translocation protein C; CtpA1 and CtpA2, C-terminal processing peptidases A 1 and 2; CYO1, Shiyou 1 (Shiyou means cotyledon in Japanese), also known as SCO2; CYP20-3, 20-kDa cyclophilin 3; CYP38, cyclophilin of $38 \mathrm{kDa}$; Cyt $b_{559}$, cytochrome $b_{559}$; Deg1; Deg2, Deg5, Deg7, and Deg8, Degradation-of-periplasmic-proteins proteases 1, 2, 5, 7, and 8; FKBP20-2, 20-kDa FK506 (tacrolimus)-binding protein; ELIP1 and ELIP2, early light-induced proteins 1 and 2; FtsH1, FtsH2, FtsH5, FtsH6, FtsH8, FtsH9, FtsH11, and FtsH12, filamentation temperature sensitive proteins $\mathrm{H} \mathrm{1,} \mathrm{2,} \mathrm{5,} \mathrm{6,} \mathrm{8,}$ 9, 11, and 12; FtsH2 and FtsH5 are also known as VAR2 and VAR1, respectively; fug1, fu-gaeri1; GTPase, GTP hydrolase; HCF106, HCF136, HCF173, HCF243, and HCF244, High Chlorophyll Fluorescence 106, 136, 173, 243, and 244; HHL1, Hypersensitive to High Light 1; HLIP, high-light-induced protein; LHCII, lightharvesting complex II; LHCB1, LHCB2, LHCB3, LHCB4, LHCB5, and LHCB6, Photosystem II light-harvesting chlorophyll $a / b$-binding proteins $1,2,3,4,5$, and 6; LHCP, light-harvesting chlorophyll a/b-binding protein; LIL3:1 and LIL3:2, light-harvesting-like proteins 3:1 and 3:2; LMM, low molecular mass; LPA1, LPA2, LPA3, and LPA19, Low Photosystem II Accumulation 1, 2, 3, and 19; LPA1 is also known as PratA; LQY1, Low Quantum Yield of Photosystem II 1; LTO1, Lumen Thiol Oxidoreductase 1; MET1, Mesophyll-Enriched Thylakoid protein 1; MPH1, Maintenance of Photosystem II under High light 1; OEC, oxygen-evolving complex; OHP1 and OHP2, one-helix proteins 1 and 2; PAM68, Photosynthesis Affected Mutant 68; PBCP, Photosystem II core phosphatase; PBF1, Photosystem Biogenesis Factor 1; pD1, precursor D1; PDI6, Protein Disulfide Isomerase 6, also known as PDIL1-2; PDIase, protein disulfide isomerase; PDIL1-2, Protein Disulfide Isomerase-Like 1-2, also known as PDI6; PPH1, Protein Phosphatase 1; PPIase, Peptidylprolyl Isomerase; PPL1 and PPL2, PsbP-Like proteins 1 and 2; PratA, Processing-associated tetratricopeptide repeat protein A, also known as LPA1; PSI, Photosystem I; PSII, Photosystem II; Psa, Photosystem II; PsaH and PsaK, Photosystem I proteins $\mathrm{H}$ and K; Psb, Photosystem II; PSB28, PSB29, and PSB33, Photosystem II proteins 28, 29 and 33; PSB29 is also known as THF1; PsbA, PsbB, PsbC, PsbD, PsbE, PsbF, PsbH, PsbI, PsbJ, PsbK, PsbL, PsbM, PsbN, PsbO, PsbP, PsbQ, PsbR, PsbT, PsbU, PsbV, PsbW, PsbX, PsbY, PsbZ, Photosystem II protein A, B, C, D, E, F, H, I, J, K, L, M, N, O, P, Q, $\mathrm{R}, \mathrm{T}, \mathrm{U}, \mathrm{V}, \mathrm{W}, \mathrm{X}, \mathrm{Y}$ and Z; PsbA, PsbB, PsbC, and PsbD are also known as D1, CP47, CP43, and D2; PsbE and PsbF are also known as cytochrome $b_{559}$ subunit alpha and beta, respectively; PsbN is also known as PBF1; PsbO, PsbP, and PsbQ are also known as the 33-, 23-, and 17-kDa protein of the oxygenevolving complex, respectively; PsbTc, chloroplast-encoded Photosystem II protein T; PsbTn, nuclear-encoded PSII protein T; RC, minimal reaction-center complex which lacks CP47 and CP43; RC47a and RC 47b, reaction-center complexes that contain CP47 but lack CP43; RBD1, rubredoxin 1; RNAi, RNA interference; SCO2, Snowy Cotyledon 2, also known as CYO1; SCP, small chlorophyll-bindinglike protein; SDR, short-chain dehydrogenase/reductase; SRP, signal recognition particle; STN7 and STN8, state transition 7 and 8; ROC4, rotamase cyclophilin 4; SEP3.1 and SEP3.2, stress-enhanced protein 3.1 and 3.2; TerC, Tellurite-resistance protein C; T-DNA, transfer DNA; TAP38, Thylakoid-Associated Phosphatase of 38 kDa; THA4, Thylakoid Assembly 4; THF1, Thylakoid Formation 1, also known as PSB29; TLP18.3 and TLP40, Thylakoid Lumen Proteins of 18.3 and $40 \mathrm{kDa}$; TPR, tetratricopeptide repeat; TRX, thioredoxin; VAR1 and VAR2, Yellow Variegated 1 and 2, also known as FtsH5 and FtsH2, respectively; VIPP1, Vesicle-Inducing Protein in Plastids 1; YCF48, hypothetical chloroplast reading frame number 48.
PsbL, PsbM, PsbR, PsbTc (chloroplast-encoded PSII protein T), PsbTn (nuclear-encoded PSII protein T), PsbW, PsbX, PsbY, and PsbZ (Nickelsen and Rengstl, 2013). Due to the loss of PsbU and PsbV during green plant evolution, cyanobacterial OEC has PsbO, PsbP, PsbQ, PsbU, and PsbV subunits but land plant OEC only contains PsbO, PsbP, and PsbQ subunits (Thornton et al., 2004; Bricker et al., 2012). The PSII-light-harvesting antenna in cyanobacteria is made of phycobilisomes, which are attached to the cytoplasmic side of PSII (Liu et al., 2005). The PSII-lightharvesting antenna (i.e., light-harvesting complex II, abbreviated as LHCII) in land plants is an integral membrane complex. LHCII contains three major trimeric PSII light-harvesting chlorophyll $a / b$-binding (LHCB) proteins LHCB1, LHCB2, and LHCB3 and three minor monomeric LHCB proteins LHCB4, LHCB5, and LHCB6 (Jansson, 1999; Liu et al., 2004). In addition to PSII subunits, more than 60 auxiliary proteins or enzymes have been found to be involved in the assembly, stability, and repair of PSII complexes (Nixon et al., 2010; Nickelsen and Rengstl, 2013; Järvi et al., 2015). This article focuses on the identification and roles of different types of proteins that influence the assembly, stability, and repair of PSII in the higher plant Arabidopsis thaliana.

\section{DE NOVO ASSEMBLY OF PSII}

De novo (Latin for "anew" or "from the beginning") PSII assembly is a sequential and highly coordinated process. The principal steps were revealed by the use of radioactive pulsechase experiments, two-dimensional blue native/sodium dodecyl sulfate-polyacrylamide gel electrophoresis, and subsequent proteomics and mass spectrometry analysis (Aro et al., 2005; Rokka et al., 2005; Boehm et al., 2012a). De novo PSII assembly in higher plants include: (1) assembly of the precursor D1PsbI (pD1-PsbI) and D2-cytochrome $b_{559}$ (D2-Cyt $b_{559}$ ) precomplexes, (2) assembly of the minimal reaction-center complex (RC), which lacks CP47 and CP43, (3) assembly of the reaction-center complex (RC47a) that contains $\mathrm{CP} 47$ but lacks CP43, (4) incorporation of LMM subunits, such as PsbH, PsbM, PsbT, and PsbR, to form RC47b, (5) incorporation of CP43, along with LMM subunit PsbK, to form the OEC-less PSII monomer, (6) assembly of the OEC and additional LMM subunits, such as PsbW and PsbZ, to form the PSII core monomer, and (7) dimerization and formation of the PSII-LHCII supercomplex (Figure 1; Rokka et al., 2005; Nixon et al., 2010; Komenda et al., 2012a; Nickelsen and Rengstl, 2013). A similar pathway exists in cyanobacteria, algae, and lower plants, suggesting that the core components of PSII and the assembly process of PSII complexes are conserved (Nixon et al., 2010; Komenda et al., 2012a; Nickelsen and Rengstl, 2013). From cyanobacteria to green algae to land plants, the initial assembly steps of photosynthetic complexes appear to be spatially separated from sites of active photosynthesis (Nickelsen and Rengstl, 2013). For instance, in Chlamydomonas reinhardtii, initial steps of de novo PSII assembly occur in discrete regions near the pyrenoid, called translation zones (Uniacke and Zerges, 2007). 


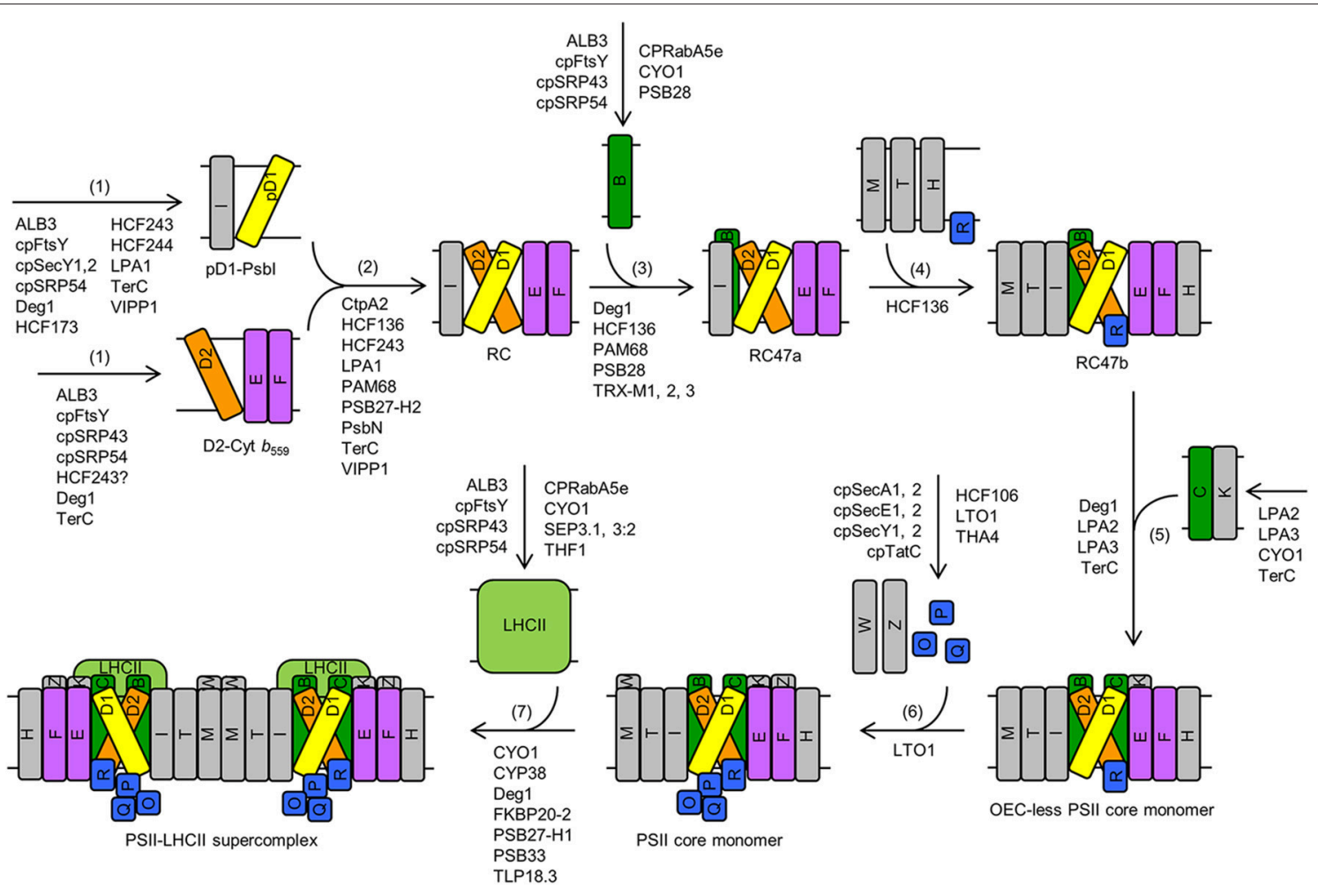

FIGURE 1 | De novo assembly of PSII in Arabidopsis. The major steps include: (1) assembly of precursor D1-Psbl (pD1-Psbl) and D2-cytochrome $b_{559}$ (D2-Cyt $b_{559}$ ) precomplexes, (2) assembly of the minimal reaction-center complex (RC), which lacks CP47 and CP43, (3) assembly of the reaction-center complex (RC47a) that contains CP47 but lacks CP43, (4) incorporation of LMM subunits, such as PsbH, PsbM, PsbT, and PsbR, to form RC47b, (5) incorporation of CP43, along with LMM subunit PsbK, to form the OEC-less PSIl core monomer, (6) assembly of the oxygen-evolving complex (OEC) and additional LMM subunits, such as PsbW and PsbZ, to form the PSII core monomer, and (7) dimerization and formation of the PSII-light-harvesting complex II (LHCII) supercomplex. Proteins that are involved in these steps are listed. Although RBD1 promotes PSII assembly and/or PSII stability, it is not depicted in this figure because it is not clear which step(s) of de novo PSII assembly this protein is involved in. Letters (B, C, D1, D2, E, F, H, I, K, M, O, P, Q, R, T, W, Z) in rectangles represent PSIl proteins PsbB (i.e., CP47), PsbC (i.e., CP43), D1, D2, PsbE, PsbF, PsbH, Psbl, PsbK, PsbM, PsbO, PsbP, PsbQ, PsbR, PsbT, PsbW, and PsbZ, respectively. Abbreviations: D2-Cyt b559, D2-cytochrome b559 precomplex; LHCII, light-harvesting complex II; OEC, oxygen-evolving complex; pD1, precursor D1; pD1-Psbl, precursor D1-Psbl precomplex; PSII, Photosystem II; RC, PSII minimal reaction-center complex; RC47a, PSII reaction-center complex with CP47, without PsbM, PsbH, PsbT, or PsbR; RC47b, PSII reaction-center complex with CP47, PsbM, PsbH, PsbT, and PsbR. For simplicity, only one name is shown for proteins with multiple names (e.g., "THF1" for THF1/PSB29).

Assembly of LHCII is recently thought to initiate on the chloroplast envelope in developing chloroplasts and on thylakoid membranes in developed chloroplasts (Tanz et al., 2012; Khan et al., 2013). LHCII assembly on the chloroplast envelope consists of four major steps: (1) partial insertion of LHCP (light-harvesting chlorophyll $a / b$-binding protein) apoproteins into the inner chloroplast envelope, (2) binding of chlorophyll to reach a stable conformation in the membrane, (3) insertion of the rest of the protein domains, and (4) further pigment binding and protein assembly into a fully assembled pigment-protein complex (Hoober et al., 2007; Dall'Osto et al., 2015). The pigment-protein complexes on the inner chloroplast envelope can be transferred to thylakoid membranes via the chloroplast-vesicle-transport system, the primary source of lipids and proteins for developing thylakoids in young chloroplasts (Tanz et al., 2012; Khan et al., 2013; Karim et al., 2014). In developed chloroplasts, LHCP proteins are primarily transported and integrated into thylakoid membranes via the chloroplast signal recognition particle (cpSRP) pathway (Cline and Dabney-Smith, 2008; Albiniak et al., 2012; Dall'Osto et al., 2015).

\section{DAMAGE, REPAIR, AND REASSEMBLY OF PSII}

The PSII repair cycle is a sequential process as well. The major steps in higher plants include: (1) high-lightinduced phosphorylation, damage, and disassembly of the PSII-LHCII supercomplex and the PSII core dimer in grana stacks, (2) lateral migration of the PSII core monomer to stroma-exposed thylakoid membranes, (35) dephosphorylation, partial disassembly of the PSII core monomer, and degradation of photodamaged D1, (6) synthesis and reassembly of new D1, (7) re-incorporation of CP43, (8) re-attachment of OEC, (9) migration of the PSII core monomer back to grana stacks, and (10) dimerization into PSII core dimers and reformation of PSII-LHCII 

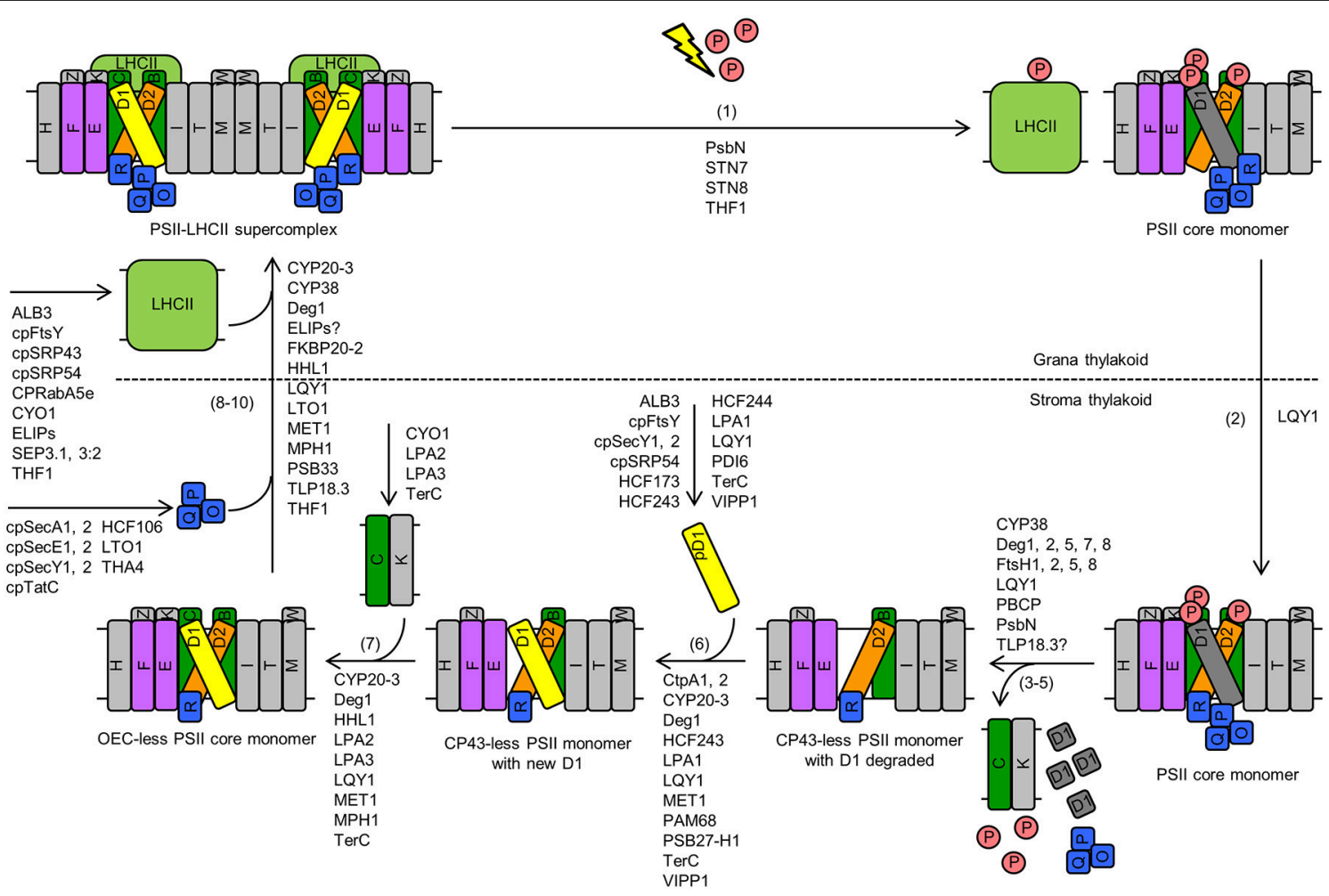

FIGURE 2 | Damage, repair, and reassembly of PSII in Arabidopsis. The major steps include: (1) high-light-induced phosphorylation, damage, and disassembly of the PSII-LHCII supercomplex and the PSII core dimer in grana stacks, (2) lateral migration of the PSII core monomer to stroma-exposed thylakoid membranes, (3-5) dephosphorylation, partial disassembly of the PSII core monomer, and degradation of photodamaged D1, (6) synthesis and reassembly of new D1, (7)

re-incorporation of CP43, (8) reattachment of OEC, (9) migration of the PSII core monomer back to grana stacks, and (10) dimerization into PSII core dimers and reformation of PSII-LHCII supercomplexes. Proteins that are involved in these steps are listed. Although PPL1 might be involved in PSII repair, it is not depicted in this figure because it is not clear which step(s) of PSII repair this protein is involved in. Letters (B, C, D1, D2, E, F, H, I, K, M, O, P, Q, R, T, W, Z) in rectangles represent PSII proteins PsbB (i.e., CP47), PsbC (i.e., CP43), D1, D2, PsbE, PsbF, PsbH, Psbl, PsbK, PsbM, PsbO, PsbP, PsbQ, PsbR, PsbT, PsbW, and PsbZ, respectively. The letter $\mathrm{P}$ in a circle represents phosphate. The yellow lightning bolt represents light. Abbreviations: LHCII, light-harvesting complex II; OEC, oxygen-evolving complex; pD1, precursor D1; PSII, Photosystem II. For simplicity, only one name is shown for proteins with multiple names (e.g., "THF1" for THF1/PSB29).

supercomplexes (Figure 2; Mulo et al., 2008; Järvi et al., 2015).

\section{PROTEINS THAT INFLUENCE THE ASSEMBLY, STABILITY, AND REPAIR OF PSII}

Assistance from a series of protein factors is required for the assembly, stability, and repair of PSII (Figures 1, 2; Mulo et al., 2008; Nickelsen and Rengstl, 2013; Järvi et al., 2015). The types of protein factors include: (1) components of thylakoid-protein-targeting complexes; (2) components of the chloroplast-vesicle-transport system, (3) PSII subunit-like proteins, e.g., PsbP-like and LHCP-like proteins, (4) atypical short-chain dehydrogenase/reductase (SDR) family proteins, (5) C-terminal D1 processing endopeptidases, (6) tetratricopeptide repeat (TPR) proteins, (7) thiol/disulfide-modulating proteins, (8) peptidylprolyl isomerases (PPIases), (9) protein kinases, (10) protein phosphatases, (11) FtsH (filamentation temperature sensitive protein $\mathrm{H}$ ) proteases, (12) Deg (Degradation of periplasmic proteins) proteases, and (13) other auxiliary proteins with unique or unknown domain compositions (Table 1). As discussed below, many proteins that are involved in de novo PSII assembly also play roles in the repair and reassembly cycle of PSII.

Some of these factors, such as components of the thylakoid-protein-targeting complexes and the chloroplastvesicle-transport system, are not specific for the assembly, stability, or repair of PSII. However, because thylakoid protein targeting and chloroplast vesicle transport are essential for translocation and accumulation of thylakoid membrane/lumen proteins, and because most of PSII subunits and assembly, stability, and repair factors are thylakoid membrane/lumen proteins, these two types of protein factors are included in this article.

\section{Components of Thylakoid-Protein-Targeting Complexes}

Four thylakoid transport and integration pathways have been identified to date (Cline and Dabney-Smith, 2008; Albiniak et al., 2012). The cPSRP pathway and an unusual pathway that 


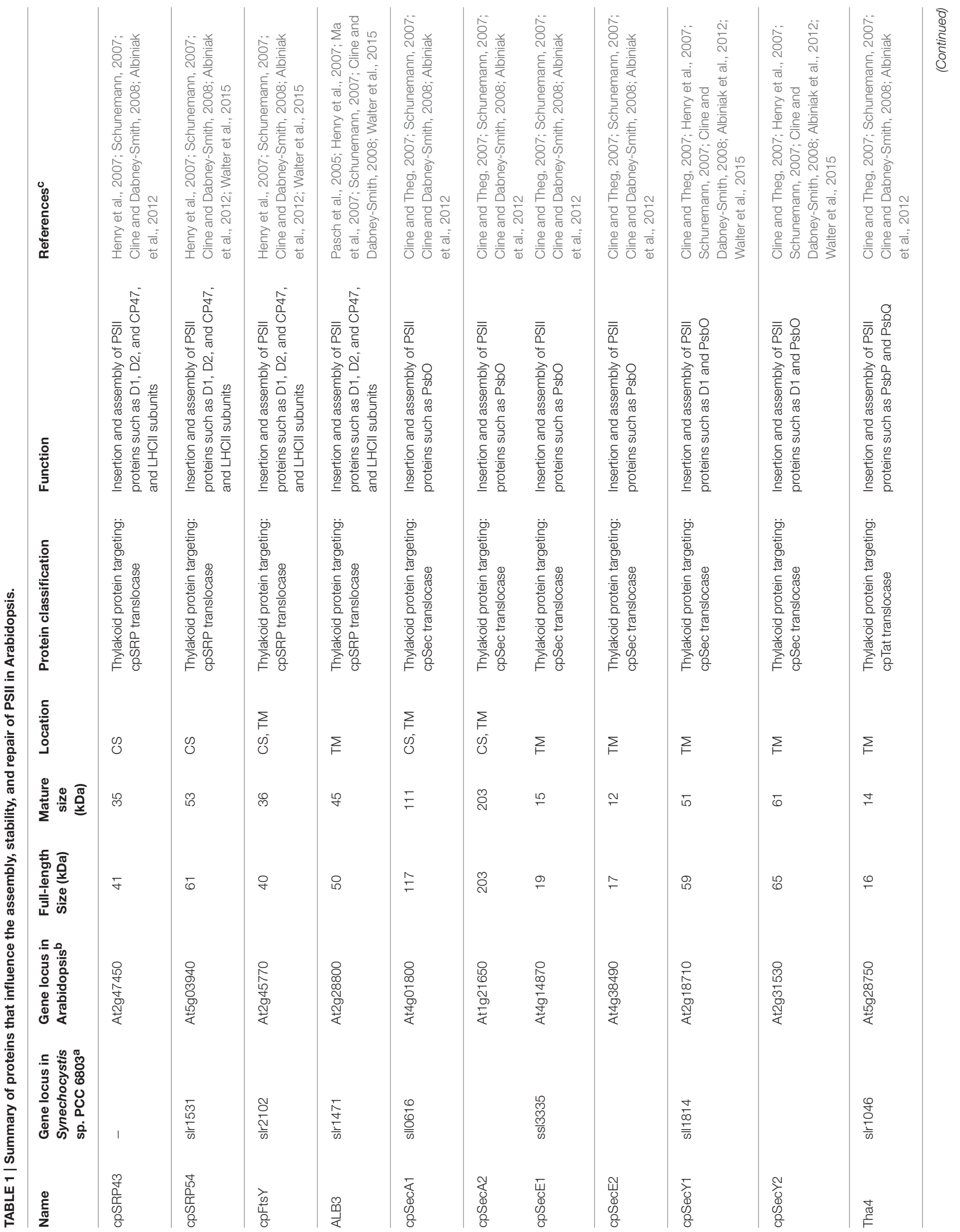




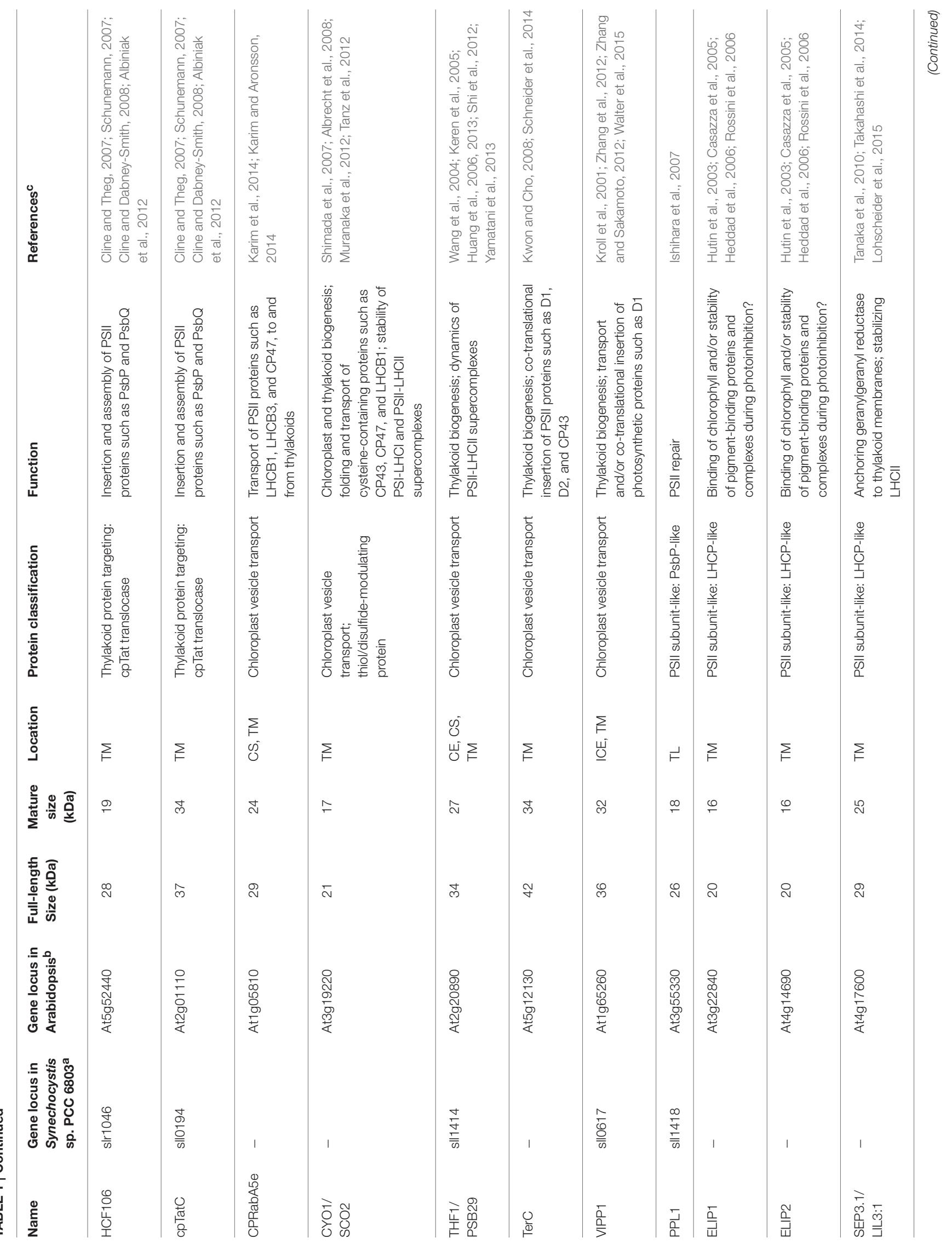




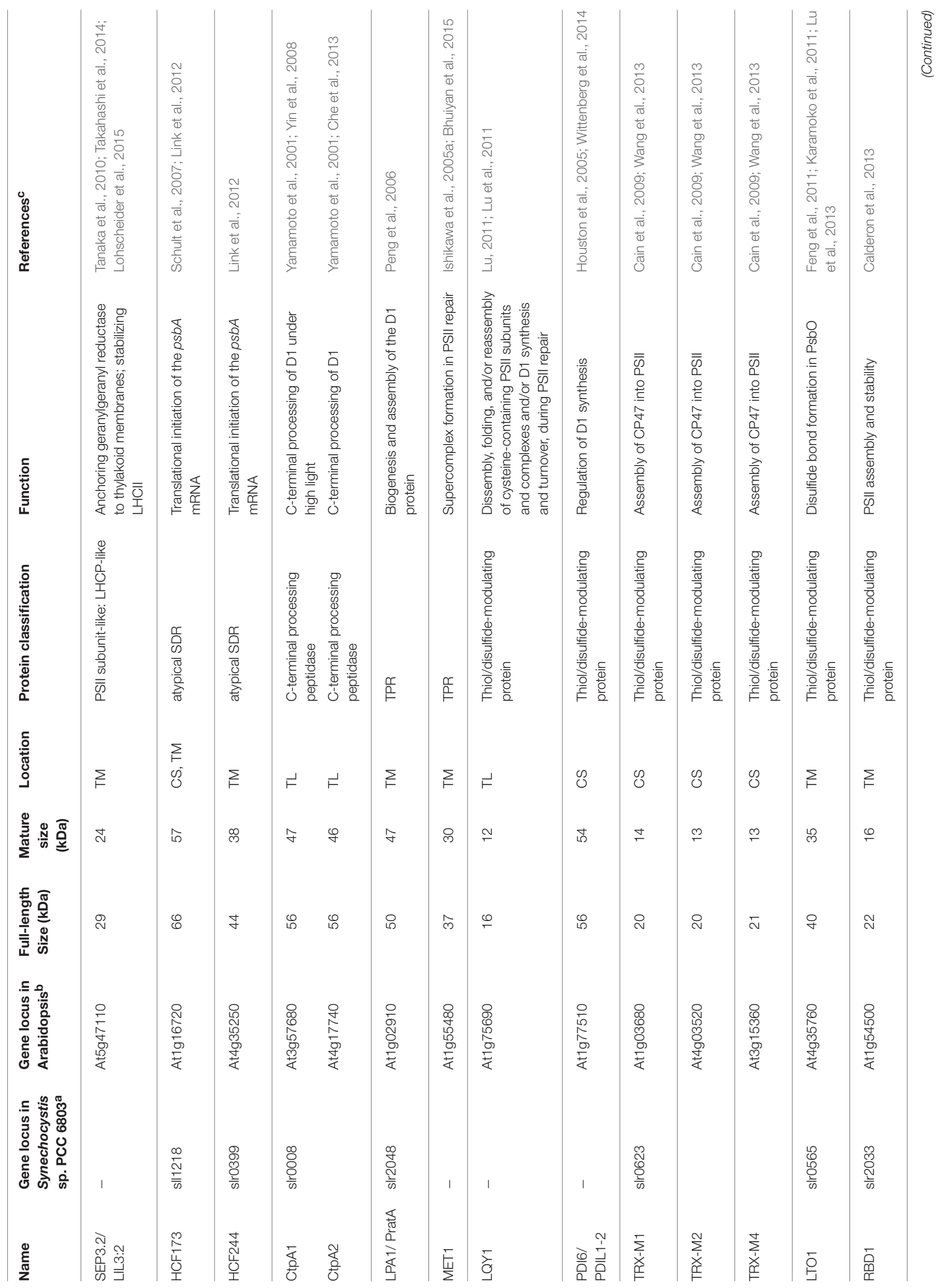




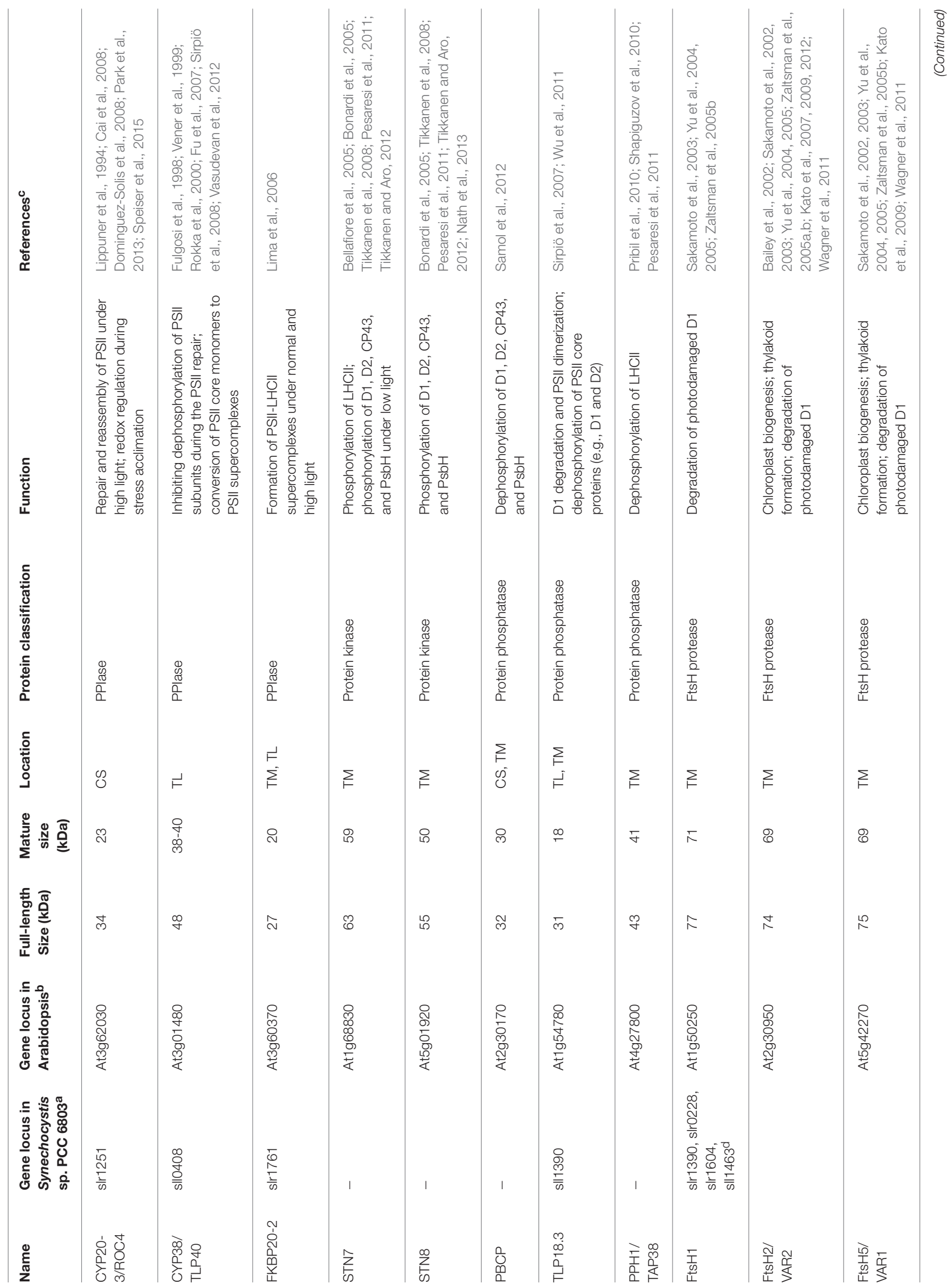




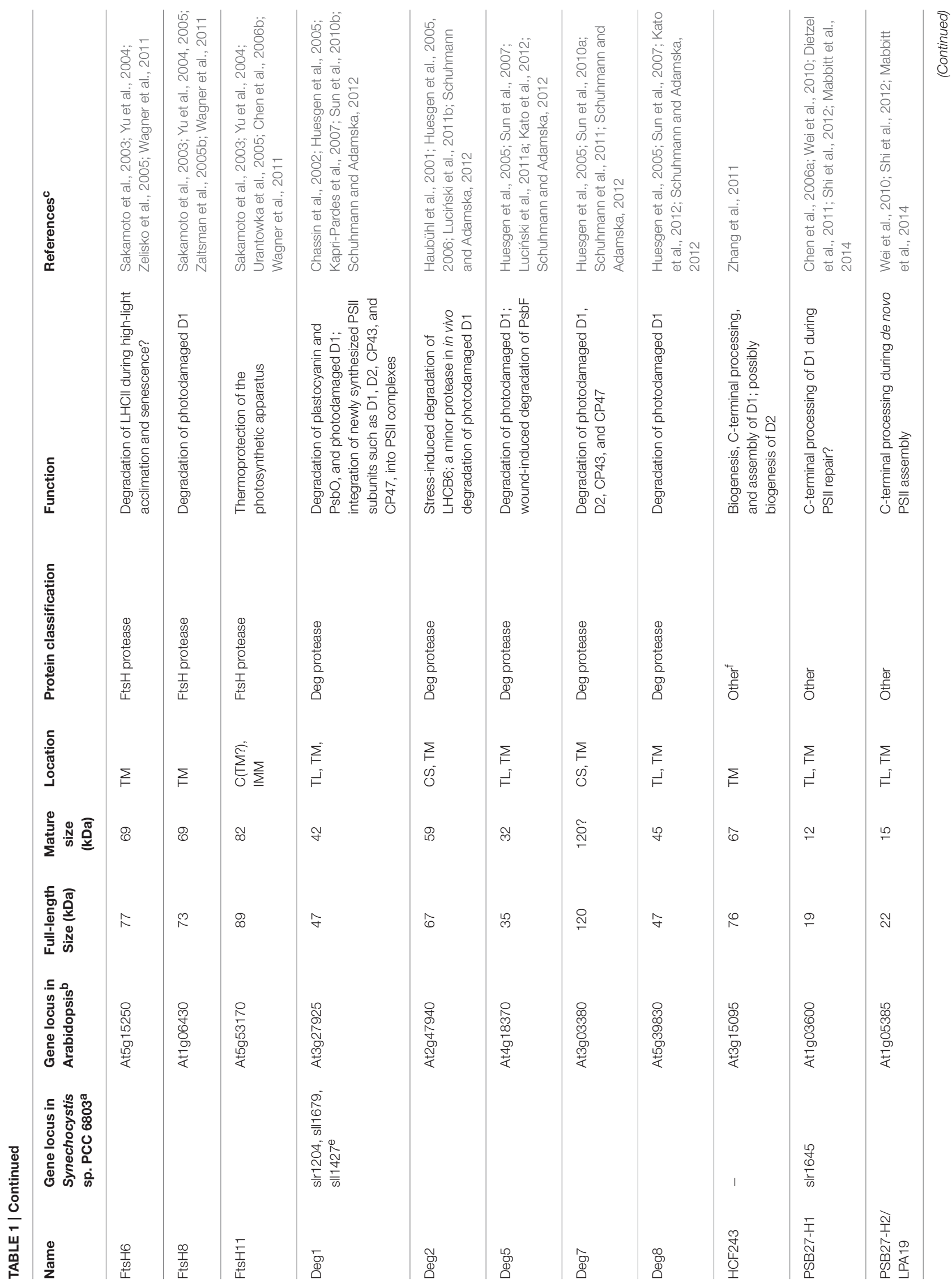




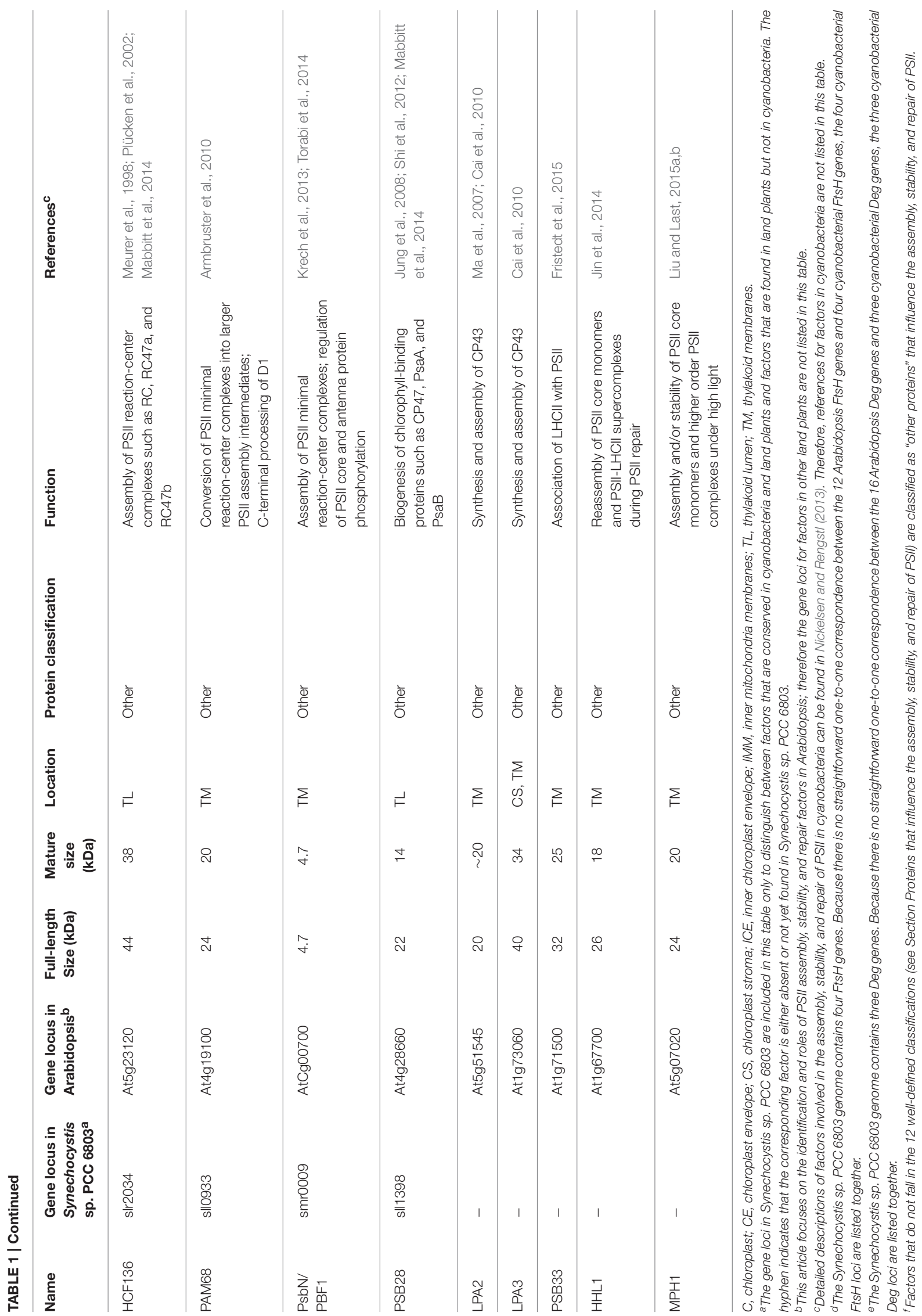


requires none of the known targeting apparatus are responsible for translocating thylakoid membrane proteins. LHCPs are translocated via the cpSRP pathway that requires the action of cpSRP43 (chloroplast Signal Recognition Particle protein of $43 \mathrm{kDa}$ ), cpSRP54 (cpSRP protein of $54 \mathrm{kDa}$ ), cpFtsY (chloroplast filamentation temperature sensitive protein $\mathrm{Y}$ ), and ALB3 (Albino3) (Figures 1, 2). ALB3 was found to interact with PSII subunits D1, D2, and CP47 (Ossenbühl et al., 2004; Pasch et al., 2005; Göhre et al., 2006), consistent with the function of the cpSRP pathway in translocating PSII proteins such as D1, D2, and CP47 (Figures 1, 2). Most of the remaining thylakoid membrane proteins are inserted by the unusual pathway that requires none of the known targeting apparatus. The cpSecAcpSecYE (cpSec means chloroplast secretory) pathway and the chloroplast twin-arginine translocation (cpTat) pathway are responsible for translocation of lumenal proteins. In Arabidopsis, each cpSec component is encoded by two genes (Table 1). The cpTat pathway has three components: Thylakoid Assembly 4 (THA4), High Chlorophyll Fluorescence 106 (HCF106), and cpTat protein C (cpTatC) (Albiniak et al., 2012). PsbO and plastocyanin are substrates of the cpSec pathway while PsbP and PsbQ are substrates of the cpTat pathway (Figures 1, 2; Albiniak et al., 2012). Like their bacterial counterparts, components of the cpSRP and cpSec pathways may act in a modular fashion (Cline and Theg, 2007; Henry et al., 2007). For instance, cpSRP54 was identified in co-translational D1 insertion intermediates, along with cpSecY (chloroplast secretory translocase $\mathrm{Y}$ ) and chloroplast ribosomes (Cline and Theg, 2007; Henry et al., 2007). Consistent with this finding, Walter et al. (2015) reported that cpSecY forms a complex with VIPP1 (Vesicle-Inducing Protein in Plastids 1) and cpSec components ALB3, cpFtsY, and cpSRP54 during co-translational integration of D1 (Figures 1, 2). How different thylakoid membrane and lumenal proteins, including components of PSII complexes, are transported and integrated into thylakoids by the above-mentioned thylakoidprotein-targeting complexes can be found in a number of reviews (Schunemann, 2007; Cline and Dabney-Smith, 2008; Albiniak et al., 2012).

\section{Components of the Putative Chloroplast-Vesicle-Transport System}

PSII is located in the thylakoid membranes of oxygenic photosynthetic organisms; therefore thylakoid membrane biogenesis is essential to PSII assembly (Nickelsen and Zerges, 2013; Rast et al., 2015). It has been suggested that thylakoids could develop from invaginations of the inner envelope membrane via a vesicle-based transfer process and these vesicles are thought as a method of transporting lipids and proteins to and from thylakoids (Hoober et al., 1991; Westphal et al., 2001, 2003; Charuvi et al., 2012; Karim and Aronsson, 2014; Rast et al., 2015). Chloroplast vesicles are typically not observed when vesicular transport from the inner chloroplast envelope to thylakoids is continuous at ambient temperature (Morré et al., 1991). When vesicular transport is blocked by low temperature, chloroplast vesicles accumulate (Morré et al., 1991). A number of proteins have been implicated as part of the chloroplast-vesicle-transport system, including CPRabA5e (chloroplast Rab GTPase A5e), CYO1/SCO2 (Shiyou 1/Snowy Cotyledon 2), THF1/PSB29 (Thylakoid Formation 1/PSII protein 29), TerC (Tellurite-resistance protein C), and VIPP1 (Karim and Aronsson, 2014; Rast et al., 2015).

CPRabA5e is a small Rab GTPase targeted to the chloroplast stroma and thylakoid membranes (Karim et al., 2014). Transfer DNA (T-DNA) insertions in the CPRabA5e gene cause a reduced amount of grana thylakoids in Arabidopsis leaves (Karim et al., 2014). After pre-incubation at $4^{\circ} \mathrm{C}$, the cprabA5e mutants have larger plastoglobules and an increased number of small vesicles, compared to the wild type. CPRabA5e was found to interact with a number of photosynthetic proteins, including PSI subunits $\mathrm{H} 2$ and $\mathrm{K}$ (PsaH2 and PsaK; Psa stands for Photosystem I), the PSII core subunit CP47, and LHCB proteins LHCB1 and LHCB3 (Karim et al., 2014). These findings led to the hypothesis that CPRabA5e is involved in transport of photosynthetic proteins, such as LHCB1, LHCB3, and CP47 (Figures 1, 2), to developing thylakoids in young chloroplasts via vesicles (Karim et al., 2014).

CYO1/SCO2 is a zinc-finger-domain-containing thylakoid membrane protein with protein disulfide isomerase (PDI) activity (Shimada et al., 2007; Albrecht et al., 2008; Muranaka et al., 2012; Tanz et al., 2012). A loss-of-function mutation in the $C Y O 1 / S C O 2$ gene results in globularly or normally shaped plastids with very big vesicles in Arabidopsis cotyledons (Tanz et al., 2012). A closer look at normally shaped chloroplasts from mutant cotyledons showed that small vesicles emerged from the inner chloroplast envelope, even at ambient temperature. These vesicles were not observed in chloroplasts from wildtype cotyledons used in the same study. These observations suggest that vesicular transport from the inner chloroplast envelope to developing thylakoids is blocked in the cotyledons of mutant seedlings and that the chloroplast-vesicle-transport system is important for thylakoid biogenesis rather than damage or programmed degradation of the thylakoid membrane system. CYO1/SCO2 was found to interact with PSI core subunits PsaA and PsaB, PSII core subunits CP43 and CP47, and LHCB1 (Muranaka et al., 2012; Tanz et al., 2012) and to co-migrate with Photosystem I-light-harvesting complex I (PSI-LHCI) and PSII-LHCII supercomplexes in blue native-polyacrylamide gel electrophoresis (BN-PAGE) (Shimada et al., 2007). Therefore, it is conceivable that $\mathrm{CYO} 1 / \mathrm{SCO} 2$ participates in transport of photosynthetic proteins, such as CP43, CP47, and LHCB1 (Figures 1, 2), to and from thylakoids via chloroplast vesicles in cotyledons (Tanz et al., 2012). The association of CYO1/SCO2 with PSI-LHCI and PSII-LHCII supercomplexes also begs the question whether this protein has a role in stabilizing these supercomplexes (Figures 1, 2).

THF1/PSB29 is a coiled-coil-domain-containing protein targeted to the chloroplast envelope, chloroplast stroma, and thylakoid membranes (Wang et al., 2004; Huang et al., 2006). The thf1/psb29 knockout mutant of Arabidopsis has a variegated phenotype in cotyledons and true leaves and the chloroplasts in the yellow sectors of thf1/psb29 leaves lack normal thylakoids and accumulate chloroplast vesicles (Wang et al., 2004; Keren et al., 2005; Zhang et al., 2009). These data led to the hypothesis that THF1/PSB29 is required for organizing chloroplast vesicles 
into mature thylakoids (Wang et al., 2004). THF1/PSB29 was thought to be involved in maintaining levels of FtsHs in plants because D1, a substrate of FtsHs, was found to be more stable in the thf1/psb29 mutant than in the wild type (Zhang et al., 2009). However, it was later found that the stay-green phenotype of the thf1/psb29 mutant is not due to reduced FtsH protease activity, because ftsh2/var2 (var2 stands for yellow variegated 2) leaves turn yellow much faster than wild-type and thf1/psb29 leaves during dark-induced senescence (Huang et al., 2013). THF1/PSB29 was found to interact with all six LHCB proteins (Huang et al., 2013); therefore it is possible that LHCB proteins are transported to thylakoid membranes via direct interaction with THF1/PSB29 (Figures 1, 2). In addition, it was proposed that THF1/PSB29 regulates the dynamics of PSII-LHCII supercomplexes during high-light stress and leaf senescence (Figure 2; Huang et al., 2013; Yamatani et al., 2013). The THF1/PSB29-deficient mutants of Arabidopsis and rice (Oryza sativa) also have a stay-green phenotype in pathogeninfected and dark-induced senescent leaves (Huang et al., 2013; Yamatani et al., 2013). In dark- and high-light-treated thf1/psb29 Arabidopsis leaves, PSII-LHCII supercomplexes are highly unstable but a type of PSII-LHCII megacomplexes is retained (Huang et al., 2013). Consistent with a role in regulating the dynamics of PSII-LHCII supercomplexes, THF1/PSB29 was found to co-migrate with trimeric and monomeric LHCII in BN-PAGE (Huang et al., 2013).

TerC is an integral thylakoid membrane protein with eight transmembrane helices (Kwon and Cho, 2008; Schneider et al., 2014). T-DNA insertions in the TerC gene caused a pigmentdeficient and seedling-lethal phenotype in Arabidopsis (Kwon and Cho, 2008; Schneider et al., 2014). This is accompanied with a substantial reduction or complete loss of thylakoid membranes and over-accumulation of chloroplast vesicles. Therefore, TerC was considered to be invloved in thylakoid biogenesis and vesicle transport (Kwon and Cho, 2008). To further analyze the function of TerC, Schneider et al. (2014) generated an artifical microRNA-based knockdown allele amiR-TerC in Arabidopsis and found that the severe phenotype of the T-DNA mutants is likely due to the subsantaially reduced rates of synthesis and insertion of PSII proteins. In line with these observations, TerC was found to interact with PSII proteins D1, D2, and CP43 as well as PSII assembly factors ALB3, LPA1/PratA (Low PSII Accumulation 1/Processing-associated tetratricopeptide repeat protein A), LPA2 (Low PSII Accumulation 2), and PAM68 (Photosynthesis Affected Mutant 68) (Schneider et al., 2014). Taken together, it is reasonable to propose that TerC is also invloved in co-translational insertion of PSII proteins (e.g., D1, D2, and CP43) into thylakoid membranes, in colaboration with other PSII assembly factors (Figures 1, 2; Schneider et al., 2014).

VIPP1 is homologous to the phage shock protein A in Escherichia coli, which is induced under various stress environments (Karim and Aronsson, 2014). VIPP1 is targeted to the inner chloroplast envelope and thylakoid membranes (Kroll et al., 2001). The vipp1 knockdown and knockout mutants of Arabidopsis are pigment-deficient and semi-lethal (i.e., unable to grow photoautotrophically) (Kroll et al., 2001; Zhang et al.,
2012; Zhang and Sakamoto, 2012). Chloroplasts from the vipp1 knockout and knockdown mutants are defective in thylakoid membrane formation and vesicle budding from inner envelope membranes. In addition, vipp1 mutant chloroplasts are swollen due to damage in the chloroplast envelope and increases in the osmotic pressure in the chloroplast stroma (Zhang et al., 2012; Zhang and Sakamoto, 2012). VIPP1 was recently found in cotranslational D1 insertion intermediates isolated from thylakoid membranes of pea (Pisum sativum) leaves, along with cpSecY, ALB3, cpFtsY, and cpSRP54 (Walter et al., 2015). Therefore, VIPP1 was proposed as a multifunctional protein that is involved in chloroplast vesicular transport, thylakoid biogenesis, and cotranslational insertion of photosynthetic proteins (Figure 1).

\section{PSII Subunit-Like Proteins, e.g., PsbP-Like and LHCP-Like Proteins}

Five PSII subunit-like proteins, including one PsbP-like protein and four LHCP-like proteins, have been implicated to be involved in the assembly, stability, and/or repair of PSII complexes or subunits. PsbP-like proteins are in the same family as PsbP proteins (Bricker et al., 2013; Ifuku, 2014). Unlike PsbP proteins, whose primary function is water splitting and oxygen evolution, PsbP-like proteins are not part of the OEC. Arabidopsis has two PsbP-like proteins: PPL1 and PPL2 (Table 1). A T-DNA insertion in the PPL1 gene led to increased sensitivity to high light and delayed recovery after photoinhibition (Ishihara et al., 2007). These data suggest that PPL1 is required for efficient repair of photodamaged PSII. Although PPL1 has not been shown to be associated with PSII in higher plants, its cyanobacterial homolog cyanoP (ssl1418) has been shown to be loosely associated with PSII and possess the same beta-sandwich fold and a wellconserved zinc-binding site as PsbP in higher plants (Ishikawa et al., 2005b; Summerfield et al., 2005; Michoux et al., 2010, 2014; Jackson et al., 2012). Further studies are needed to investigate the precise function of PPL1 in PSII repair. Unlike PPL1, PPL2 is required for the accumulation of chloroplast $\mathrm{NAD}(\mathrm{P}) \mathrm{H}$ dehydrogenase complex (Ishihara et al., 2007); therefore the function of PPL2 is not discussed in this review.

LHCP-like proteins are in the same superfamily as LHCPs (Heddad et al., 2012). Unlike LHCPs, whose primary function is light-harvesting, most LHCP-like proteins are involved in chlorophyll- and carotenoid-binding, assembly and stability of chlorophyll-protein complexes, and/or photoprotection. LHCPlike proteins can be classified into three subfamilies: three-helix, early light-induced proteins (ELIPs), two-helix, stress-enhanced proteins (SEPs), and one-helix, high-light-induced, small chlorophyll-binding-like proteins (OHPs/HLIPs/SCPs). ELIPs are restricted to green algae and land plants, SEPs are ubiquitously present in photosynthetic eukaryotes, OHP1 exists in cyanophages, cyanobacteria, and photosynthetic eukaryotes, and OHP2 exists in eukaryotes. The Arabidopsis genome encodes two ELIPs, six SEPs, and two OHPs (Heddad et al., 2012).

ELIPs appear to be associated with PSII under standard conditions in pea plants; they become associated with monomeric and trimeric LHCII under high light in Arabidopsis 
(Adamska and Kloppstech, 1991; Heddad et al., 2006). Heddad et al. (2006) showed that the relative amounts of ELIP transcripts and proteins increase as the light intensity increases. It was therefore proposed that ELIPs may be involved in photoprotection by binding free chlorophyll released during degradation of pigment-binding proteins or by stabilizing the assembly of pigment-binding proteins during photoinhibition (Adamska and Kloppstech, 1991; Hutin et al., 2003). The potential function of ELIPs in stabilizing LHCII and PSII-LHCII supercomplexes under light stress is included in Figure 2. However, elip1 and elip2 single knockout mutants and the elip1 elip2 double knockout mutant have a similar phenotype and light sensitivity as the wild-type Arabidopsis plants (Casazza et al., 2005; Rossini et al., 2006). Additional studies are needed to dissect the exact functions of ELIP proteins.

Among the six SEPs in Arabidopsis, the functions of SEP3.1/LIL3:1 (SEP stands for stress-enhanced protein; LIL stands for light-harvesting-like protein) and SEP3.2/LIL3:2 have been extensively studied (Tanaka et al., 2010; Takahashi et al., 2014; Lohscheider et al., 2015; Mork-Jansson et al., 2015). The anti-sense sep3.1/lil3:1 mutant and the sep3.1/lil3:1 sep3.2/lil3:2 double knockout mutant are deficient in chlorophyll and $\alpha$-tocopherol biosynthesis (Tanaka et al., 2010; Lohscheider et al., 2015). The deficiency is due to a substantial reduction in the amount of chlorophyll and $\alpha$-tocopherol biosynthetic enzyme geranylgeranyl reductase. In line with these findings, SEP3.1/LIL3:1 and SEP3.2/LIL3:2 were found to interact with geranylgeranyl reductase and their transmembrane domain was found to be important for the interaction (Tanaka et al., 2010; Takahashi et al., 2014). Therefore, SEP3.1/LIL3:1 and SEP3.2/LIL3:2 were proposed to be involved in chlorophyll and tocopherol biosynthesis by anchoring and stabilizing geranylgeranyl reductase to thylakoid membranes. In addition, SEP3.1/LIL3:1 and SEP3.2/LIL3:2 were found to accumulate with increasing light irradiance and they are associated with subcomplexes of LHCII (Lohscheider et al., 2015). Thus, it is also possible that SEP3.1/LIL3:1 and SEP3.2/LIL3:2 may function in stabilizing LHCII (Figures 1, 2).

The OHP genes have an expression pattern similar to the ELIP and SEP genes, whose expression is up-regulated upon high light (Mulo et al., 2008; Heddad et al., 2012). The function of OHP1 is not yet known, but OHP2 was showed to be associated with PSI under low or high light and was therefore proposed to play a role in photoprotection of PSI (Andersson et al., 2003). Recently, a cyanobacterial OHP family protein was found to bind chlorophyll $a$ and $\beta$-carotene and possess an energy-dissipative conformation, suggesting that OHP family proteins may have a photoprotective role (Staleva et al., 2015).

\section{Atypical SDR Family Proteins}

Classic SDR family proteins have an intact cofactor-binding site (TGXXGXXG) and an intact catalytic tetrad (NSYK), which are required for their SDR activity (Persson et al., 2009). Unlike classic SDR family proteins, atypical SDR family proteins have no known enzyme activity because they have an altered glycinerich cofactor-binding site and partially or completely lack the signature catalytic tetrad (Link et al., 2012). Two atypical SDR family proteins have been found to be important for PSII: HCF173 (High Chlorophyll Fluorescence 173) and HCF244 (High Chlorophyll Fluorescence 244) (Schult et al., 2007; Link et al., 2012; Chidgey et al., 2014; Knoppová et al., 2014). Compared to HCF244, HCF173 is 200 amino acids longer and its SDR domain is fragmented into two regions. HCF173 and HCF244 have the same subcellular localization: they are both predominantly associated with chloroplast membranes, with a small fraction located in the chloroplast stroma. Loss-of-function mutations in the HCF173 or HCF244 gene result in similar defects in Arabidopsis: a drastic reduction in D1 synthesis, inability to accumulate PSII subunits, substantial decreases in PSII activity, and a complete loss of photoautotrophy (Schult et al., 2007; Link et al., 2012). The hcf173 and hcf244 single mutants are able to grow on sucrose-supplemented media but they are pale green and much smaller than the wild type (Link et al., 2012). Polysome association experiments demonstrated that these defects are caused by reduced translation initiation of the $p s b A$ transcript (Schult et al., 2007; Link et al., 2012). The decrease in translation initiation is accompanied by a reduction in $p s b A$ mRNA stability. The $h c f 173 h c f 244$ double mutant grown on sucrose-supplemented media is smaller than the single mutants, suggesting that simultaneous loss of HCF173 and HCF244 has an additive effect (Link et al., 2012). The function of HCF173 and HCF244 in translation initiation of the psbA transcript is included in Figures 1, 2. Some SDR family proteins, such as dihydrolipoamide acetyltransferases, glyceraldehyde3-phosphate dehydrogenase, and lactate dehydrogenase, have evolved the capacity to bind RNA (Hentze, 1994; Nagy et al., 2000; Pioli et al., 2002; Bohne et al., 2013). Therefore, it is possible that HCF173 and HCF244 may act as RNA-binding proteins and facilitate translation initiation of the $p s b A$ mRNA (Link et al., 2012).

\section{C-terminal Processing Peptidases}

The PSII reaction-center protein D1 is often synthesized in the precursor form ( $\mathrm{pD} 1$ ), with a C-terminal extension of 8-16 amino acids (Nixon et al., 1992; Anbudurai et al., 1994; Liao et al., 2000). In plants, this C-terminal extension is cleaved in a single step by C-terminal processing peptidase A (CtpA) at an early step of de novo PSII assembly or at the reassembly step of PSII repair (Figures 1, 2). CtpAs are serine endopeptidases with a serine/lysine catalytic dyad (Anbudurai et al., 1994; Liao et al., 2000; Yamamoto et al., 2001). Recombinant spinach (Spinacia oleracea) CtpA exhibited efficient proteolytic activity toward thylakoid membrane-embedded pD1 (Yamamoto et al., 2001). Arabidopsis has three CtpAs in the thylakoid lumen and the functions of CtpA1 and CtpA2 have been studied (Yin et al., 2008; Che et al., 2013).

Under normal growth conditions, T-DNA insertions in the Arabidopsis CtpA1 gene do not cause changes to plant growth and morphology, PSII activity, or thylakoid membrane complex formation (Yin et al., 2008). Under high light, the ctpA1 mutant displays retarded growth, accelerated D1 turnover, as well as increased photosensitivity and delayed recovery of PSII activity. Therefore, CtpA1 was proposed to be involved in D1 protein C-terminal processing in the PSII repair cycle (Figure 2). 
Unlike the ctpA1 mutant, the T-DNA mutant of the Arabidopsis CtpA2 gene is lethal under normal light but is viable in sucrose-supplemented media under low light (Che et al., 2013). The viable ctpA2 mutant displays a complete loss of the mature D1 protein, reduced levels of other PSII core proteins, a severely decreased level of PSII supercomplexes, and a substantial reduction or complete loss of PSII activity. pD1 and other PSII subunits in the viable ctpA2 mutant are present in PSII monomers and PSII dimers but absent in PSII supercomplexes. These data suggest that CtpA2 is indispensible for C-terminal processing of D1 (Figure 1), which in itself is essential for de novo PSII assembly. A weak allele expressing $~ 2 \%$ of the wildtype level of CtpA2 appears to be normal under normal light but displays stunted growth and over-accumulation of pD1 under elevated light (Che et al., 2013). These data suggest that CtpA2 is also involved in C-terminal processing of D1 during high-lightinduced PSII repair (Figure 2).

\section{TPR Proteins}

The TPR is a 34-amino acid repeated motif that ubiquitously exits among all organisms (Ishikawa et al., 2005a). Two TPR proteins have been found to be involved in PSII assembly and/or repair: LPA1/PratA and MET1 (Mesophyll-Enriched Thylakoid protein 1) (Ishikawa et al., 2005a; Peng et al., 2006; Bhuiyan et al., 2015).

LPA1/PratA is an intrinsic thylakoid membrane protein with two tandem TPR motifs and a double-pass transmembrane domain (Klinkert et al., 2004; Peng et al., 2006). T-DNA insertions in the Arabidopsis LPA1/PratA gene result in reduced growth, pale-green leaves, reduced PSII activity, reduced amounts of PSII proteins, reduced synthesis of D1 and D2, increased turnover of PSII core subunits D1, D2, CP43, and CP47, and inefficient assembly of PSII (Peng et al., 2006). The transcript levels of genes encoding PSII core subunits are unchanged in the mutants. LPA1/PratA was found to directly interact with D1 in a split-ubiquitin yeast-two-hybrid assay. These data suggest that LPA1/PratA has a role in biogenesis and assembly of D1 (Figures 1, 2). Consistent with this hypothesis, LPA1/PratA was identified in thylakoidmembrane-associated ribosome nascent chain fractions (Peng et al., 2006).

MET1 has an N-terminal PDZ domain and a C-terminal TPR motif, which are conserved across green algae and land plants (Ishikawa et al., 2005a; Bhuiyan et al., 2015). MET1 is peripherally attached to thylakoid membranes on the stromal side and it is enriched in stroma lamellae (Bhuiyan et al., 2015). T-DNA insertions in the Arabidopsis MET1 gene do not cause obvious changes to the accumulation and assembly state of the photosynthetic apparatus under normal light (Bhuiyan et al., 2015). Under fluctuating light, the met1 mutants demonstrate reduced growth, decreased PSII efficiency, a near-complete loss of PSII-LHCII supercomplexes, and increased amounts of unassembled CP43. Loss of MET1 also causes increased photosensitivity of PSII activity and an accelerated rate of D1 turnover under high light. MET1 was found to co-migrate with a series of PSII subcomplexes, such as PSII dimers, PSII core monomers, CP43-less PSII monomers, and PSII reactioncenter complexes (i.e., RC, RC47a, and RC47b), in BN-PAGE. Therefore, MET1 was proposed to be involved in supercomplex formation during PSII repair (Figure 2). In line with this hypothesis, MET1 was found to interact with the stromal loops of PSII core subunits CP43 and CP47 (Bhuiyan et al., 2015).

\section{Thiol/Disulfide-Modulating Proteins}

Thiol/disulfide modulation is important for regulating photosynthetic processes (Järvi et al., 2013; Karamoko et al., 2013). Three types of thiol/disulfide-modulating proteins have been found to be involved in the assembly, stability, function, and repair of PSII: protein disulfide isomerases (PDIases), protein disulfide reducing proteins, and protein thiol oxidizing proteins.

As mentioned above, thylakoid membrane protein CYO1/SCO2 was found to play a role in chloroplast and thylakoid biogenesis and vesicular transport of photosynthetic proteins to developing thylakoids in cotyledons (Shimada et al., 2007; Albrecht et al., 2008; Tanz et al., 2012). CYO1/SCO2 has a $\mathrm{C}_{4}$-type zinc-finger domain with two conserved CXXCXGXG repeats, the signature domain for PDIase activity (Shimada et al., 2007). Recombinant CYO1/SCO2 is able to catalyze reduction of protein disulfide bonds and oxidative renaturation of reduced and denatured protein substrates, indicating that CYO1/SCO 2 is a PDIase (Shimada et al., 2007). CYO1/SCO2 was found to interact with PsaA, PsaB, CP47, CP43, and LHCB1 (Muranaka et al., 2012; Tanz et al., 2012). These five CYO1/SCO2-interacting proteins contain cysteine in the hydrophobic region(s). Therefore, CYO1/SCO2 may also participate in folding of cysteine-containing PSI and PSII subunits (Figures 1, 2), by forming transient disulfide bonds with its protein substrates via the CXXC motif (Jessop et al., 2007; Feige and Hendershot, 2011).

LQY1 (Low Quantum Yield of PSII 1) is another thylakoid membrane protein with PDIase activity. Full-length LQY1 has a chloroplast transit peptide, a transmembrane domain, and a C-terminal $\mathrm{C}_{4}$-type zinc-finger domain with four conserved CXXCXGXG repeats ( $\mathrm{Lu}$ et al., 2011). Recombinant LQY1 is able to catalyze oxidative renaturation of reduced and denatured protein substrates and reductive renaturation of oxidized protein substrates. T-DNA insertions in the Arabidopsis LQY1 gene cause reduced efficiency of PSII photochemistry, increased sensitivity to high light, and increased accumulation of reactive oxygen species under high light. The lqy1 mutants were found to accumulate fewer PSII-LHCII supercomplexes and have altered rates of high-light-induced D1 turnover and re-synthesis. The lqy1 mutant phenotype can be suppressed by complementation of lqy1 mutants with the wild-type LQY1 gene (Lu, 2011). LQY1 is associated with the PSII core monomer and the CP43less PSII monomer (a marker for ongoing PSII repair and reassembly) and it is most abundant in stroma-exposed thylakoid membranes, where important steps of PSII repair occurs (Lu et al., 2011). Under high light, LQY1 associated with PSII monomers increases at the expense of free LQY1 and LQY1 associated with smaller PSII complexes. Immunoprecipitation analysis showed that LQY1 interacts with PSII core subunits 
CP47 and CP43, which contain three and four conserved cysteine residuals, respectively. Therefore, it was proposed that LQY1 is involved in PSII repair ( $\mathrm{Lu}, 2011$; Lu et al., 2011). It is possible that LQY1 participates in dissembly, folding, and/or reassembly of cysteine-containing PSII subunits and complexes and/or regulates D1 synthesis and turnover during PSII repair (Figure 2). These hypotheses require further investigation.

PDI6/PDIL1-2 (Protein Disulfide Isomerase 6/Protein Disulfide Isomerase-Like 1-2) contains two redox-active thioredoxin domains (with the WCGHC active site), two redox-inactive thioredoxin-like domains, and a C-terminal endoplasmic reticulum retention signal KDEL (Houston et al., 2005; Wittenberg et al., 2014). PDI6/PDIL1-2 is dual-targeted to chloroplasts and the endoplasmic reticulum; chloroplasttargeted PDI6 is located in the stroma (Wittenberg et al., 2014). Similar to CYO1/SCO2 and LQY1, recombinant PDI6/PDIL1-2 is capable of catalyzing oxidative renaturation of reduced and denatured protein substrates. Compared to wild-type Arabidopsis, the pdi6-1 and pdi6-2 knockdown mutants display increased resistance to high light, reduced photoinhibition, and an accelerated rate of D1 synthesis (Wittenberg et al., 2014). Therefore, it was proposed that PDI6/PDIL1-2 may function as an attenuator of D1 synthesis during PSII repair (Figure 2).

Thioredoxins are small proteins that contain a redox-active thioredoxin domain with the WCGHC active site (Cain et al., 2009). As enzymes, thioredoxins are active in the reduced form and are able to reduce disulfide bonds in protein substrates (Cain et al., 2009). Thioredoxins are important for regulating thiol/disulfide homeostasis inside chloroplasts (Cain et al., 2009). Three M-type thioredoxins (TRX-M1, M2, and M4) have been found to be involved in PSII biogenesis in chloroplasts (Wang et al., 2013). TRX-M1, M2, and M4 are associated with minor PSII assembly intermediate subcomplexes and they interact with PSII core subunits D1, D2, and CP47. Simultaneous inactivation of the three Arabidopsis TRX-M genes causes pale-green leaves, reduced PSII activity, decreased accumulation of PSII complexes, and increased accumulation of reactive oxygen species. PSII core proteins D1 and CP47 were found to be able to form redox-sensitive intermolecular disulfide bonds and concurrent loss of the three M-type thioredoxins interrupts the redox status of these PSII core subunits. According to these results, Wang et al. (2013) proposed that the three TRX-M proteins may assist incorporation of CP47 into PSII core complexes (Figure 1).

In addition to PDIases and thioredoxins, a new type of proteins has been found to regulate thiol/disulfide homeostasis and they mainly act as oxidases by converting free thiols on protein substrates into disulfide bridges. One example is Lumen Thiol Oxidoreductase 1 (LTO1), a thylakoid membrane protein with an integral-membrane vitamin $\mathrm{K}$ epoxide reductase domain and a soluble disulfide-bond A oxidoreductase-like domain (Feng et al., 2011; Karamoko et al., 2011; Lu et al., 2013). Each of the two domains contains four conserved cysteine residues (a pair of cysteine residues in the CXXC motif and another pair of separate cysteine residues), which are critical for the disulfide-bond-forming activity of LTO1 (Feng et al., 2011). According to membrane topology analysis, Feng et al. (2011) proposed that the eight conserved cysteine residues are positioned on the lumenal side of thylakoid membranes. This led to the hypothesis that LTO1 is involved in formation of the intramolecular disulfide bond in PsbO (Figures 1, 2), which is located on the lumenal side of thylakoid membranes (Karamoko et al., 2011). Consistent with this hypothesis, LTO1 was found to interact with PsbO1 and PsbO2 and catalyze formation of intramolecular disulfide bonds in recombinant PsbO (Karamoko et al., 2011). In line with these observations, the amounts of PsbO, PsbQ, and PsbQ are substantially reduced in the LTO1deficient Arabidopsis mutants and the mutants display reduced efficiency of PSII photochemistry, increased accumulation of reactive oxygen species, a smaller plant size, and delayed growth (Karamoko et al., 2011; Lu et al., 2013).

RBD1 (rubredoxin 1) is a small iron-containing protein with a C-terminal transmembrane domain and a rubredoxin domain with two redox-active CXXC motifs (Calderon et al., 2013). Homologs of RBD1 have been found in thylakoid membranes but not plasma membranes of cyanobacteria and in thylakoid membranes of green algae and land plants (Shen et al., 2002; Calderon et al., 2013). The rbd1 knockout mutants in the cyanobacterium Synechocystis sp. PCC 6803, the green alga Chlamydomonas reinhardtii, and the higher plant Arabidopsis display a substantial reduction or complete loss of PSII activity and photoautotrophy (Calderon et al., 2013). The amounts of PSII core subunits, such as D1, D2, and CP47, are reduced by $40-90 \%$ in these mutants while other components of the photosynthetic apparatus, such as PSI, cytochrome $b_{6} f$ complex, and ATP synthase, are not affected. Based on these data, Calderon et al. (2013) proposed that RBD1 is required for the assembly and/or stability of PSII in oxygenic photosynthetic organisms. Further studies are needed to dissect the precise function of RBD1.

\section{PPlases}

Peptide bonds to proline have cis and trans conformations (Fischer et al., 1998; Ingelsson et al., 2009). Therefore, folding of proteins such as PSII subunits often involves cis-trans proline isomerization, which is catalyzed by PPIases (He et al., 2004; Romano et al., 2004; Ingelsson et al., 2009). Three PPIase families have been established according to their immunosuppressant ligand specificity: cyclophilins (CYPs), FK506 (tacrolimus)binding proteins (FKBPs), and parvulins (Fischer et al., 1998; He et al., 2004; Ingelsson et al., 2009). Two CYPs and one FKBP have been found to be important for the assembly, stability, and/or repair of PSII (Järvi et al., 2015).

CYP20-3/ROC4 (20-kDa cyclophilin 3/rotamase cyclophilin 4) is localized in the chloroplast stroma (Lippuner et al., 1994). The Arabidopsis T-DNA insertion mutant of CYP203/ROC4 has a normal phenotype and normal PSII function under ambient light (Cai et al., 2008). Under high light, PSII in the cyp20-3/roc4 mutant exhibits increased photosensitivity and delayed recovery, which caused growth retardation and leaf yellowing. Under high light, D1 degradation is not affected in the mutant but repair and reassembly of photodamaged PSII is impaired. According to the cyp20-3/roc4 mutant phenotype, Cai et al. (2008) proposed that CYP20-3/ROC4 is involved in repair and reassembly of PSII under high light (Figure 2). The 
PPIase activity of CYP20-3/ROC4 makes it a good candidate for catalyzing correct folding of PSII proteins during the repair process of PSII. CYP20-3/ROC4 was also reported to link light and redox signals to cysteine biosynthesis and stress acclimation (Dominguez-Solis et al., 2008; Park et al., 2013; Speiser et al., 2015).

CYP38/TLP40 (cyclophilin of $38 \mathrm{kDa} /$ Thylakoid Lumen Protein of $40 \mathrm{kDa}$ ) is predominantly confined in the lumen of non-appressed thylakoids (Fulgosi et al., 1998). Full-length CYP38/TLP40 has a bipartite thylakoid lumen targeting transit peptide, a leucine zipper, a phosphatase-binding module, an acid region for protein-protein interaction, and a C-terminal cyclophilin-type PPIase domain (Fulgosi et al., 1998; Sirpiö et al., 2008). CYP38/TLP40 isolated from spinach leaves demonstrates in vivo PPIase activity and co-purification with a thylakoid membrane phosphatase (Fulgosi et al., 1998; Vener et al., 1999). In addition, it was reported that CYP38/TLP40 could be released from thylakoid membranes to the thylakoid lumen upon heat stress and the release is associated with activation of dephosphorylation of PSII subunits in thylakoid membranes (Rokka et al., 2000). Therefore, it was proposed that CYP38/TLP40 acts as a phosphatase inhibitor and regulates dephosphorylation of PSII subunits during PSII repair (Figure 2). Consistent with this hypothesis, T-DNA insertions in the Arabidopsis CYP38/TLP40 gene cause increased phosphorylation of PSII core subunits and increased photosensitivity of PSII activity (Fu et al., 2007; Sirpiö et al., 2008). In addition, CYP38/TLP40 was found to interact with CP47 through its cyclophilin domain (Vasudevan et al., 2012). Dephosphorylation of PSII subunits during light acclimation is carried out by a type $2 \mathrm{C}$ protein phosphatase called PSII core phosphatase (PBCP) (Samol et al., 2012). Further studies are needed to investigate whether CYP38/TLP40 interacts with $\mathrm{PBCP}$ and inhibits its phosphatase activity. The cyp38/tlp40 mutants display retarded growth, palegreen leaves, increased accumulation of PSII monomers, and decreased accumulation of PSII supercomplexes, even under low or ambient light (Fu et al., 2007; Sirpiö et al., 2008). In addition, CYP38/TLP40 was found to co-migrate with PSII core monomers in BN-PAGE (Sirpiö et al., 2008). Therefore, it is likely that CYP38 also functions in conversion of PSII core monomers into higher order PSII complexes (Figures 1, 2).

FKBP20-2 (20-kDa FK506-binding protein 2) is located in the thylakoid lumen (Lima et al., 2006). Recombinant FKBP202 demonstrates PPIase activity and the C-terminus of FKBP202 has a unique pair of cysteine residues which can be reduced by thioredoxin. T-DNA insertions in the Arabidopsis FKBP202 gene cause smaller plant sizes, reduced chlorophyll contents, stunted growth, reduced PSII activity, increased accumulation of PSII monomers and PSII dimers, and decreased accumulation of PSII supercomplexes under normal light conditions (Lima et al., 2006). Under higher light, the difference in PSII activity between the mutant and the wild type is more pronounced. According to the mutant phenotype, Lima et al. (2006) proposed that FKBP202 functions in formation of PSII-LHCII supercomplexes under normal and high light (Figures 1, 2).

\section{Protein Kinases}

Multiple studies have shown that high light induces phosphorylation of PSII core proteins, such as D1, D2, CP43, and PsbH (Rintamaki et al., 1997; Vener et al., 2001), which facilitates migration of photodamaged PSII complexes from grana stacks to stroma lamellae (Tikkanen et al., 2008; Goral et al., 2010). Two serine/threonine protein kinases were found to be localized the thylakoid membranes: STN7 (state transition 7) and STN8 (state transition 8) (Bellafiore et al., 2005; Bonardi et al., 2005; Nath et al., 2013). Light-induced phosphorylation of PSII core proteins is carried out by STN8, and to a lesser degree under low light also by STN7 (Figure 2; Bonardi et al., 2005; Tikkanen et al., 2008; Nath et al., 2013). The primary role of STN7 is phosphorylation of LHCII proteins, which leads to displacement of LHCII from PSII to PSI (Bellafiore et al., 2005). Phosphorylation of PSII core proteins promotes unfolding of grana stacks and migration of photodamaged PSII complexes from grana stacks to stroma-exposed thylakoids. This allows easier access of membrane or membrane-associated proteases and co-translational integration of D1 and therefore facilitates repair of photodamaged PSII complexes and proteins (Bonardi et al., 2005; Tikkanen et al., 2008; Khatoon et al., 2009; Goral et al., 2010; Herbstová et al., 2012; Tikkanen and Aro, 2012).

\section{Protein Phosphatases}

While the migration of photodamaged PSII complexes is assisted by phosphorylation of PSII core proteins, dephosphorylation of D1 is necessary for efficient turnover of photodamaged D1 in stroma lamellae (Järvi et al., 2015). Two chloroplast protein phosphatases, PBCP and TLP18.3 (Thylakoid Lumen Protein of $18.3 \mathrm{kDa}$ ), have demonstrated in vivo or in vitro phosphatase activity toward PSII core proteins (Sirpiö et al., 2007; Wu et al., 2011; Samol et al., 2012). PBCP is a type 2C protein phosphatase predominantly found in the chloroplast stroma, with a minor fraction associated with thylakoid membranes (Samol et al., 2012). Compared to wild-type Arabidopsis, the PBCP-deficient mutants display delayed dephosphorylation of PSII core proteins (D1, D2, CP43, and PsbH) and normal dephosphorylation of LHCII proteins, upon exposure to far-red light, which favors PSI excitation and dephosphorylation of thylakoid proteins (Samol et al., 2012). Samol et al. (2012) concluded that PBCP is required for efficient dephosphorylation of PSII core proteins (Figure 2).

TLP18.3 is a thylakoid membrane protein with the Nterminal domain of unknown function located in the thylakoid lumen (Sirpiö et al., 2007). It was originally identified as an auxiliary protein involved in dimerization of PSII monomers and degradation of photodamaged D1 (Figures 1, 2). The TLP18.3-deficient Arabidopsis mutants do not show a clear visual phenotype under normal growth conditions but exhibit retarded growth under fluctuating light (Sirpiö et al., 2007), suggesting that TLP18.3 is more important to PSII repair than to de novo PSII assembly. Compared to the wild type, the TLP18.3-deficient mutants have fewer PSII dimers and more PSI monomers under normal and fluctuating light. In addition, the rate of high-light-induced D1 turnover is $\sim 50 \%$ slower in 
the TLP18.3-deficient mutants. Consistent with its dual roles in dimerization of PSII monomers, which occurs in grana stacks, and degradation of photodamaged D1, which takes place in stroma lamellae, TLP18.3 was found evenly distributed between grana stacks and stroma lamellae (Sirpiö et al., 2007). It was later found that the domain of unknown function in TLP18.3 possesses acid phosphatase activity toward synthetic phosphorylated oligopeptides that resemble the phosphorylation sites of PSII core proteins D1 and D2 (Wu et al., 2011). However, how the acid phosphatase activity of TLP18.3 is related to the role of TLP18.3 in PSII assembly and repair is still not clear (Figure 2): the phosphorylation sites of D1 are exposed to the stroma side while the acid phosphatase domain of TLP18.3 is located in the thylakoid lumen.

Dephosphorylation of LHCII proteins, such as LHCB1 and LHCB2, is carried out by PPH1/TAP38 (Protein Phosphatase 1/Thylakoid-Associated Phosphatase of $38 \mathrm{kDa}$ ) (Pribil et al., 2010; Shapiguzov et al., 2010). PPH1/TAP38 is a type 2C protein phosphatase with a C-terminal single-pass transmembrane domain; it is predominantly located in stroma lamellae and grana margins, where active dephosphorylation of LHCII and PSII core proteins occurs. Loss-of-function mutations in the Arabidopsis PPH1/TAP38 gene causes decreased dephosphorylation of LHCII while overexpression of PPH1/TAP38 enhances dephosphorylation of LHCII (Pribil et al., 2010; Shapiguzov et al., 2010). The phosphorylation status of PSII core proteins is largely unaffected in the $p$ ph1/tap38 mutants, suggesting that the primary function of PPH1/TAP38 is dephosphorylation of LHCII proteins. Recombinant PPH1/TAP38 is able to dephosphorylate LHCII directly, in an in vitro assay (Pribil et al., 2010). Reversible phosphorylation of LHCII is important for the movement of LHCII between PSII and PSI, according to the changes in the spectral composition of incident light. Therefore, the catalytic activities of LHCII kinase STN7 and phosphatase PPH1/TAP38 are important for balancing the light absorption capacity between PSI and PSII (Pesaresi et al., 2011).

\section{FtsH Proteases}

FtsH proteases are ubiquitous ATP-dependent, zinc metalloendopeptidases (Yu et al., 2004). FtsHs typically consist of an N-terminal double-pass transmembrane domain, an ATPase domain, and a C-terminal zinc-binding site. Crystal structures of the ATPase domain of bacterial FtsHs and single-particle electron cryo-microscopy analysis of cyanobacterial FtsHs showed that FtsHs exist as ringlike hexamers (Krzywda et al., 2002; Niwa et al., 2002; Boehm et al., 2012b). Bacteria contain one FtsH gene and the FtsH protein forms homohexamers while cyanobacteria and eukaryotes have multiple $\mathrm{FtsH}$ genes and the $\mathrm{FtsH}$ proteins form heterohexamers (Mann et al., 2000; Krzywda et al., 2002; Niwa et al., 2002; Zaltsman et al., 2005b; Boehm et al., 2012b). The Arabidopsis genome encodes $12 \mathrm{FtsH}$ proteases; eight FtsHs (FtsH1, FtsH2/VAR2, FtsH5/VAR1-FtsH9, and FtsH12; VAR1 stands for Yellow Variegated 1) were verified experimentally to be chloroplast-targeted; FtsH11 was showed to be dual targeted to the chloroplast (possible thylakoid membranes) and the inner mitochondria membrane (Chen et al., 2000; Takechi et al., 2000; Sakamoto et al., 2002, 2003; Urantowka et al., 2005).
FtsH2/VAR2 and FtsH5/VAR1 were found to be localized to thylakoid membranes, with their catalytic domain facing the stromal side of the membrane (Chen et al., 2000; Sakamoto et al., 2003). Among the nine chloroplast- or dual-targeted FtsHs, the functions of FtsH1, FtsH2/VAR2, FtsH5/VAR1, FtsH6, FtsH8, and FtsH11 have been explored experimentally. FtsH and Deg proteases have been known to be involved in degradation of photodamaged D1. Early in vitro studies suggested that this is a two-step process including the initial cleavage at the stromal DE loop via Deg2 and the subsequent removal of the N-terminal fragment by FtsHs (Lindahl et al., 1996, 2000; Spetea et al., 1999; Haubühl et al., 2001). It was later proposed that FtsHs play a more important role than Deg proteases in D1 turnover (Silva et al., 2003; Nixon et al., 2005, 2010; Huesgen et al., 2009; Kato et al., 2012; Komenda et al., 2012a).

Loss-of-function mutations in the Arabidopsis FtsH2/VAR2 or FtsH5/VAR1 gene cause variegated leaves (Sakamoto et al., 2002, 2003; Yu et al., 2004, 2005; Zaltsman et al., 2005a,b; Kato et al., 2007, 2009, 2012; Wagner et al., 2011). The green sectors in the ftsh2/var2 or ftsh5/var 1 mutants are formed by cells with normal chloroplasts and the white leaf sectors are formed by viable cells with undifferentiated plastids (Chen et al., 2000; Sakamoto et al., 2002; Kapri-Pardes et al., 2007). These data suggest that FtsH2/VAR2 and FtsH5/VAR1 are required for chloroplast biogenesis and thylakoid formation (Chen et al., 2000; Sakamoto et al., 2002; Zaltsman et al., 2005a,b; KapriPardes et al., 2007).

Compared to wild-type leaves, the green leaf sectors of the $f t s h 2 /$ var 2 or $f t s h 5 /$ var 1 mutants demonstrate increased photosensitivity and delayed recovery of PSII activity (Zaltsman et al., 2005a,b). Chloroplasts in the green sectors of the ftsh $2 /$ var2 or ftsh5/var1 mutants accumulate fewer PSII supercomplexes, more PSII subcomplexes, and more reactive oxygen species than wild-type chloroplasts (Kato et al., 2009). These observations are due to the proteolytic activity of FtsHs toward photodamaged D1 (Bailey et al., 2002; Kato et al., 2009). Because the variegated phenotype complicates biochemical analyses, Kato et al. (2009) used another mutation, fu-gaeril (fug1), to suppress leaf variegation, and generated the non-variegated ftsh2/var2 fug1 and ftsh5/var1 fug1 plants. Compared to the fug1 single mutant, photodamaged D1 is not replaced in the ftsh $2 /$ var 2 fug 1 and ftsh5/var1 fug 1 mutants, under different light intensities. Taken together, these data show that FtsH2/VAR2 and FtsH5/VAR1 play an important role at the early stage of D1 turnover and not just in the subsequent removal of D1 degradation products (Figure 2; Bailey et al., 2002; Kato et al., 2009).

The phenotypes observed in the ftsh $2 /$ var 2 or $f t s h 5 /$ var 1 mutants are absent in the ftsh 1 , ftsh 6 , ftsh 8 , and ftsh 11 single mutants under normal or high-light conditions (Sakamoto et al., 2003; Zaltsman et al., 2005b; Chen et al., 2006b; Wagner et al., 2011). These findings suggest that FtsH2/VAR2 and FtsH5/VAR1 play a dominant role in chloroplast biogenesis, thylakoid formation, and PSII repair (Sakamoto et al., 2003). Phylogenetic analysis showed that FtsH1 and FtsH5 are two duplicated genes (subunit type A), so are FtsH2 and FtsH8 (subunit type B) (Yu et al., 2004, 2005; Zaltsman et al., 2005b). 
The phenotype of the ftsh $/$ var2 mutant can be restored by overexpression of FtsH8; the ftsh2/var2 ftsh 8 double mutant is infertile; and the ftsh 1 ftsh $5 /$ varl double mutant resembles the ftsh2/var2 ftsh 8 double mutant (Yu et al., 2004; Zaltsman et al., 2005b). These data suggest that FtsH1 and FtsH5/VAR1 are interchangeable, so are FtsH2/VAR2 and FtsH8, and that the presence of two types of FtsH subunits is necessary for chloroplast biogenesis, thylakoid formation, and PSII repair (Figure 2; Zaltsman et al., 2005b).

FtsH6 was reported to participate in degradation of LHCII in Arabidopsis leaves during high-light acclimation and senescence (Zelisko et al., 2005). Using an in vitro degradation system (i.e., isolated thylakoid membranes), Zelisko et al. (2005) showed that, compared to the wild type, the ftsh 6 knockout mutant has reduced degradation of LHCB1 after high-light acclimation and reduced degradation of LHCB3 after darkinduced senescence. However, in vivo degradation of LHCII proteins does not appear to be impaired in the ftsh 6 knockout mutants (Wagner et al., 2011). Under various conditions, including high-light acclimation and dark-induced senescence, the abundances of LHCB1 and LHCB3 in the ftsh 6 knockout mutants are not statistically different from those in the wild type. Further investigation is needed to understand the precise role of FtsH6.

FtsH11 was reported to be critical in thermoprotection of the photosynthetic apparatus (Chen et al., 2006b; Wagner et al., 2011). When exposed to temperatures above $30^{\circ} \mathrm{C}$, which are permissive for wild-type Arabidopsis, the growth and development of the ftsh11 mutants is arrested (Chen et al., 2006b). Compared to the wild type under the same high-temperature treatment, the ftsh11 mutants have reduced levels of chlorophyll and reduced PSII activity. Consistent with the hypothesis that FtsH11 is involved in thermotolerance, the expression of the FtsH11 gene is up-regulated by high temperature (Chen et al., 2006b).

\section{Deg Proteases}

Deg proteases are ubiquitous ATP-independent, serine endopeptidases (Schuhmann and Adamska, 2012). The Arabidopsis genome encodes 16 Deg proteases, five of which are peripherally attached to thylakoid membranes: two (Deg2 and Deg7) on the stroma side and three (Deg1, Deg5, and Deg8) on the lumenal side (Huesgen et al., 2005; Schuhmann and Adamska, 2012). These five chloroplast-localized Deg proteases have been proposed to be involved in degradation of photodamaged D1 (Schuhmann and Adamska, 2012). In addition to the trypsin-like protease domain, most Deg proteases, such as Deg1, Deg2, Deg7, and Deg8, have at least one PDZ domain for protein-protein interactions. It is conceivable that these chloroplast-localized PDZ domain-containing Deg proteases may act as chaperones and function in assembly of the photosynthetic apparatus (Sun et al., 2010b; Schuhmann and Adamska, 2012).

Arabidopsis RNA interference (RNAi) lines of Deg1 have a smaller plant size, increased sensitivity of PSII activity to high light, and increased accumulation of non-degraded (and presumably photodamaged) D1 (Kapri-Pardes et al., 2007). The RNAi lines display decreased accumulation of the 16and $5.2-\mathrm{kDa}$ C-terminal degradation products of $\mathrm{D} 1$, which correspond to the cleavage products at the lumenal CD loop, and immediately after the transmembrane helix E, respectively. The addition of recombinant Deg1 into inside-out thylakoid membranes isolated from the Deg1-deficient plants induces formation of the 5.2-kDa C-terminal degradation product of D1. These data suggest that Deg1 is involved in degradation of photodamaged D1 (specifically, the cleavage at the lumenal $\mathrm{CD}$ loop immediately downstream of the transmembrane helix E) in PSII repair (Figure 2; Kapri-Pardes et al., 2007). Unfortunately, D1 turnover was not assessed in Deg1 RNAi lines. Deg1 is capable of degrading lumenal proteins plastocyanin and PsbO, suggesting that Deg1 may also acts as a general purpose endopeptidase in the thylakoid lumen (Chassin et al., 2002).

Because Deg1 has a PDZ domain for protein-protein interactions, Sun et al. (2010b) investigated whether Deg1 functions as a chaperone during assembly of PSII complexes (Sun et al., 2010b). Deg1 was found to co-migrate with D1 in BN-PAGE and pull down D1, D2, CP43, and CP47 in an immunoprecipitation assay. Recombinant Deg1 has the ability to fold reduced and denatured protein substrates in the presence of both reduced and oxidized glutathione. The RNAi lines of Deg1 display reduced accumulation of PSII complexes and normal accumulation of other photosynthetic complexes. In addition, assembly of newly synthesized PSII subunits into PSII dimers and PSII supercomplexes is hindered in the RNAi lines, although synthesis of the corresponding proteins in chloroplasts is not impaired. Based on these results, Sun et al. (2010b) proposed that Deg1 also acts as a chaperone and functions in the integration of newly synthesized PSII subunits, such as D1, D2, CP43, and CP47, into PSII complexes (Figures 1, 2). However, these experimental data do not rule out the possibility that Deg1 is directly involved in degradation of these PSII subunits.

Deg2 has a PDZ domain and a short hydrophobic segment, in addition to the trypsin-like protease domain (Haubühl et al., 2001). Recombinant Deg2 demonstrates proteolytic activity toward photodamaged D1 and is able to produce the $23-\mathrm{kDa}$ $\mathrm{N}$-terminal and the $10-\mathrm{kDa} \mathrm{C}$-terminal degradation products, which correspond to the cleavage products at the stromal DE loop (Haubühl et al., 2001). Although recombinant Deg2 is proteolytically active, the deg2 knockout mutants have the same plant morphology, PSII activity, and D1 turnover rate as wild-type Arabidopsis, under normal or elevated light (Huesgen et al., 2006). Therefore, it was proposed that Deg2 functions as a minor protease in in vivo degradation of photodamaged D1 (Figure 2). Deg2 was also reported to be involved in stress-induced degradation of LHCB6 (Luciński et al., 2011b).

Deg5 and Deg8 form heterohexamers and the Deg5-to-Deg8 ratio is $\sim 1: 1$ (Sun et al., 2007). Deg5 is $\sim 120$ amino acids shorter than Deg1 and Deg8 and it does not contain any PDZ domain (Schuhmann and Adamska, 2012). Although Deg8 has a PDZ domain, it shows no chaperone activity (i.e., PDIase activity) 
toward reduced and denatured protein substrates (Sun et al., 2010b). Recombinant Deg8 demonstrates proteolytic activity toward photodamaged D1 and is able to produce the $16-\mathrm{kDa} \mathrm{N}$ terminal and the $18-\mathrm{kDa} \mathrm{C}$-terminal degradation products, which correspond to the cleavage products at the lumenal CD loop (Sun et al., 2007). Although only recombinant Deg8 is proteolytically active, the deg5 and deg8 single knockout mutants of Arabidopsis both display impaired degradation of newly synthesized D1 and the impairment is more pronounced in the $\operatorname{deg} 5 \operatorname{deg} 8$ double mutant (Sun et al., 2007). The defect in D1 turnover in the mutants is reflected in PSII activity. PSII in the deg5 and deg8 single mutants exhibits increased sensitivity to high light and the sensitivity is more obvious in the deg5 deg8 double mutant. Under normal light, the two single mutants and the double mutant have a normal phenotype. Based on these data, Sun et al. (2007) proposed that Deg5 and Deg8 act as heterohexameric endopeptidases and cleave photodamaged D1 at the lumenal CD loop during PSII repair (Figure 2). Deg5 has also been reported to be involved in wounding-related disposal of PsbF (Luciński et al., 2011a).

Deg7 (1097 amino acids at full length) is twice as long as most Deg proteases; it has two trypsin-like protease domains (one active and one degenerated) and four PDZ domains (three active and one degenerated) (Schuhmann et al., 2011). The domain composition suggests that Deg7 is the result of a whole-gene duplication event followed by subsequent degeneration (Schuhmann et al., 2011). Deg7 forms homotrimers and the oligomerization is mediated through the degenerated protease domain (Schuhmann et al., 2011). Recombinant Deg7 demonstrates proteolytic activity toward photodamaged D1, D2, CP43, and CP47 (Sun et al., 2010a). Compared to wild-type Arabidopsis, the deg7 null mutant displays retarded growth, reduced PSII activity, and reduced degradation of PSII core proteins D1, D2, CP43, and CP47 under high light. However, under normal light, there is no apparent difference in plant growth or morphology between the $\operatorname{deg} 7$ mutant and the wild type. These data suggest that Deg7 is involved in cleavage of photodamaged PSII core proteins D1, D2, CP47, and CP43 from the stroma side during PSII repair (Figure 2; Sun et al., 2010a).

\section{HCF243, PSB27-H1, and PSB27-H2/LPA19 in Biogenesis, C-Terminal Processing, and/or Assembly of D1}

HCF243 (High Chlorophyll Fluorescence 243) is an intrinsic thylakoid membrane protein with no recognizable domain or motif (Zhang et al., 2011). A loss-of-function mutation in the Arabidopsis HCF243 gene causes substantial reductions in accumulation of PSII core subunits (D1, D2, CP43, and CP47) and assembly of PSII complexes (Zhang et al., 2011). Unlike PSII core subunits, the amounts of extrinsic PSII subunits, PSII antenna proteins, and other non-PSII thylakoid membrane proteins are hardly affected in the hcf243 mutant. In line with these observations, PSII activity is dramatically reduced in the $h c f 243$ mutant and the mutant has pale-green leaves and a much smaller plant size than the wild type. Pulse-labeling experiments indicated that these defects are caused by the severely reduced synthesis of D1 and to a lesser degree of D2 (Zhang et al., 2011). Indeed, the $h c f 243$ mutant over-accumulates $\mathrm{pD} 1$, the D1 precursor with an unprocessed C-terminus. HCF243 was also found to interact with D1 in vivo (Zhang et al., 2011). These data suggest that HCF243 is involved in biogenesis, processing, and assembly of D1 and possible biogenesis of D2 as well (Figures 1, 2).

PSB27s (PSII protein 27s) are thylakoid lumen proteins peripherally attached to thylakoid membranes (Chen et al., 2006a; Wei et al., 2010). Cyanobacterial Psb27 was found to facilitate assembly of the OEC manganese and plays a role in PSII repair (Nowaczyk et al., 2006; Liu et al., 2011a,b; Komenda et al., 2012b). The Arabidopsis genome encodes two PSB27s: PSB27-H1 and PSB27-H2/LPA19 (LPA19 stands for Low PSII Accumulation 19). Under normal light, the PSII protein composition is not changed and PSII activity is only slightly reduced in the Arabidopsis T-DNA insertion mutant of PSB27H1 (Chen et al., 2006a; Dietzel et al., 2011). However, the psb27H1 mutant has reduced amounts of PSII-LHCII supercomplexes, suggesting that PSB27-H1 is required for the formation and stability of PSII-LHCII supercomplexes (Figure 1; Dietzel et al., 2011). Under high light, PSII activity and the amount of D1 decrease much faster in the psb27-H1 mutant than in the wild type. In addition, the $p s b 27-H 1$ mutant displays delayed recovery of PSII activity after photoinhibition, suggesting that PSB27-H1 is involved in the repair cycle of photodamaged PSII. Unlike psb27-H1, the psb27-H2/lpa19 mutants of Arabidopsis have palegreen leaves, a smaller plant size, and reduced PSII activity even under normal light conditions (Wei et al., 2010). Pulselabeling experiments showed that C-terminal processing of D1 is impaired in the psb27-H2/lpa19 null mutants. PSB27-H2/LPA19 was found to specifically interact with the soluble C-terminus of precursor and mature D1. It was concluded that PSB27H2/LPA19 functions in C-terminal processing of D1 (Figure 1; Wei et al., 2010).

\section{HCF136, PAM68, and PsbN/PBF1 in Assembly OF PSII Reaction Complexes}

HCF136 (High Chlorophyll Fluorescence 136) is a lumenal protein found in stroma lamellae; it contains no recognizable domain or motif (Meurer et al., 1998). The cyanobacterial homolog of HCF136, YCF48 (hypothetical chloroplast reading frame number 48), was found to interact with $\mathrm{pD} 1$, and to a lesser degree, partially processed and unassembled D1, but not with mature and unassembled D1 or D2, in a split-ubiquitin yeast-two-hybrid assay (Komenda et al., 2008). In addition, higher-plant HCF136 was found to be associated with the PSII precomplex D2-Cyt $b_{559}$ and PSII reaction-center complexes RC, RC47a, and RC47b (Plücken et al., 2002). The HCF136deficient Arabidopsis mutant is able to accumulate PSI and cytochrome $b_{6} f$ complex proteins, but unable to accumulate PSII proteins, perform PSII activity, or grow photoautotrophically (Meurer et al., 1998; Plücken et al., 2002). Pulse-labeling studies showed that the $h c f 136$ mutant is defective in biogenesis of PSII minimal reaction-center complexes, not in biosynthesis 
of PSII proteins (Meurer et al., 1998; Plücken et al., 2002). Therefore, it was proposed that HCF136 is involved in assembly of PSII reaction-center complexes such as RC, RC47a, and RC47b (Figure 1). Consistent with this hypothesis, HCF136 was found to interact with another PSII assembly factor, PAM68 (Armbruster et al., 2010).

PAM68 in vascular plants is an integral thylakoid membrane protein with an acidic domain and a double-pass transmembrane domain (Armbruster et al., 2010). PAM68 was found to be associated with LMM complexes that are formed at an early step of PSII assembly (Armbruster et al., 2010). These complexes contain D1, D2, and LPA1/PratA, which may correspond to PSII minimal reaction-center complexes. PAM68 was found to interact with a number of PSII core subunits (D1, D2, CP43, CP47, PsbH, and PsbI) and PSII assembly factors (ALB3, HCF136, and LPA1/PratA, and LPA2). The PAM68-deficient Arabidopsis mutants have pale-green leaves, drastically reduced PSII activity, and severely retarded growth, under normal growth conditions. Consistent with these observations, the pam68 mutants have severely reduced amounts of PSII core subunits, and they over-accumulate PSII reaction-center complexes at the expense of higher order PSII complexes. PAM68 was therefore proposed to be necessary for converting PSII minimal reaction-center complexes into larger PSII complexes (Figure 1; Armbruster et al., 2010). The pam68 mutants were also found to over-accumulate pD1 (Armbruster et al., 2010), suggesting that PAM68 is also necessary for efficient C-terminal processing of D1 (Figures 1, 2).

PsbN is a small thylakoid membrane protein encoded by the plastid genome (Krech et al., 2013; Torabi et al., 2014). It was originally thought as a PSII subunit (Ikeuchi et al., 1989); however, two recent studies showed that PsbN is not a PSII subunit, but an assembly factor of PSII (Krech et al., 2013; Torabi et al., 2014). Thus, Krech et al. (2013) proposed a new name, Photosystem Biogenesis Factor 1 (PBF1) for this protein. Tobacco (Nicotiana tabacum) homoplastomic $\Delta p s b N / p b f 1$ mutants have pale-green leaves and slow autotrophic growth (Krech et al., 2013). Pulse-labeling and two-dimensional gel electrophoresis showed that formation of PSII precomplexes, e.g., pD1-PsbI and D2-Cyt $b_{559}$, is not affected in the $\Delta p s b N / p b f 1$ mutants but assembly of PSII minimal reaction-center complexes and higher order PSII complexes is hampered (Torabi et al., 2014). It was concluded that PsbN/PBF1 is involved in formation of PSII minimal reaction-center complexes (Figure 1). The $\Delta p s b N / p b f 1$ mutants are extremely sensitive to light, even at relatively low light and PSII in the $\Delta p s b N / p b f 1$ mutants is unable to recover from photoinhibition (Torabi et al., 2014). These data indicate that PsbN/PBF1 may also function in PSII repair, which occurs in stroma lamellae. Consistent with this hypothesis, PsbN was found to be predominantly located in stroma lamellae (Torabi et al., 2014). Loss of PsbN changes the phosphorylation status of PSII core proteins and LHCII proteins, which is important for the migration of photodamaged PSII complexes from grana stacks to stromaexposed thylakoids, and for balancing the light absorption capacity between PSI and PSII, respectively. Therefore, it is possible that $\mathrm{PsbN}$ is involved in PSII repair by regulating the phosphorylation status of PSII core proteins and LHCII proteins (Figure 2).

\section{PSB28 in Biogenesis of Chlorophyll-Binding Proteins Such as CP47, PsaA, and PsaB}

PSB28 (PSII protein 28) is a small protein predominantly localized in the stroma/cytoplasm (Shi et al., 2012). In Synechocystis sp. PCC 6803, a small fraction of Psb28 was found to be associated with unassembled CP47, RC47, and PSII monomers at the stroma side (Dobáková et al., 2009; Boehm et al., 2012a). Deletion of this protein in Synechocystis sp. PCC 6803 cause a decreased amount of unassembled CP47 and increased amounts of PSII minimal reaction-center complexes and unassembled D1 (Dobáková et al., 2009). In addition, the $\Delta p s b 28$ mutant exhibits reduced synthesis of CP47 and PsaA/PsaB heterodimers. Therefore, Psb28 was considered to function in biogenesis and assembly of chlorophyll-containing proteins such as $\mathrm{CP} 47, \mathrm{PsaA}$, and $\mathrm{PsaB}$ in cyanobacteria (Dobáková et al., 2009). Little is known about the role of PSB28 in photosynthetic eukaryotes except that PSB28 does exist in higher plants and that the absence of PSB28 results in a pale-green phenotype in rice (Jung et al., 2008; Mabbitt et al., 2014). However, because PSB28 is evolutionary conserved, it is reasonable to predict that PSB28 in higher plants may also function in biogenesis and assembly of chlorophyll-containing proteins such as CP47 (Figure 1).

\section{LPA2 and LPA3 in Synthesis and Assembly of CP43}

LPA2 is a small intrinsic thylakoid membrane protein with a Cterminal double-pass transmembrane domain (Ma et al., 2007). LPA3 (Low PSII Accumulation 3) is a chloroplast protein without any transmembrane domain or any other recognizable domain or motif. However, sub-chloroplast fractionation revealed that LPA3 could be located in chloroplast stroma or associated with thylakoid membranes (Cai et al., 2010). Although LPA2 and LPA3 are not homologous, they were identified as two auxiliary proteins assisting incorporation of CP43 into PSII via interaction with cpSRP translocase ALB3 (Ma et al., 2007; Cai et al., 2010). LPA2 and LPA3 were found to interact with CP43, ALB3, and each other (Cai et al., 2010). Pulse-labeling experiments showed that assembly from CP43-less reaction-center complexes to PSII monomers and formation of PSII-LHCII supercomplexes are distinctively slower in the lpa2 and lpa3 single mutants than in wild-type Arabidopsis (Ma et al., 2007; Cai et al., 2010). Synthesis of CP43 is greatly reduced in the lpa2 and lpa3 single mutants while synthesis of other PSII core subunits D1, D2, and CP47 is comparable between the single mutants and the wild type (Ma et al., 2007; Cai et al., 2010). LPA2 and LPA3 appear to be functional redundant because the lpa2 lpa3 double mutant has no detectable amounts of D1, D2, CP43, and CP47 and is seedling-lethal. Taken together, LPA2 and LPA3 were proposed to be involved in synthesis and assembly of CP43 (Figures 1, 2; Ma et al., 2007; Cai et al., 2010). 


\section{PSB33 in Association of LHCll with PSII}

PSB33 (PSII protein 33) is a thylakoid membrane protein with an N-terminal Rieske-type domain exposed to the stroma side, a double-pass transmembrane domain, and a C-terminal partial chlorophyll-binding domain (Fristedt et al., 2015). PSB33 was found to co-migrate with PSII-LHCII supercomplexes, PSII dimers, PSII monomers, and CP43-less PSII monomers in BNPAGE (Fristedt et al., 2015). Loss-of-function mutations in the Arabidopsis PSB33 gene cause reduced amounts of PSII-LHCII supercomplexes, and increased amounts of PSII dimers, lower state transition, lower non-photochemical quenching, increased photosensitivity, and retarded growth (Fristedt et al., 2015). According to these data, Fristedt et al. (2015) proposed that PSB33 may mediate the association of LHCII with PSII core complexes (Figure 1) and balance the light absorption capacity between PSII and PSI. It is likely that PSB33 also functions in attaching LHCII to PSII during reassembly of repaired PSII (Figure 2), because the defect in PSII activity in the psb33 mutants is more pronounced under higher light (Fristedt et al., 2015).

\section{HHL1 and MPH1 in Protection of PSII from Photodamage}

HHL1 (Hypersensitive to High Light 1) is a thylakoid membrane protein with a single-pass transmembrane domain, and a Cterminal partial von Willebrand factor type A domain, which is known to mediate protein-protein interactions (Jin et al., 2014). Under high light, the HHL1-deficient mutants have lower efficiency of PSII photochemistry, lower amounts of PSII core subunits and PSII-LHCII supercomplexes, and higher amounts of reactive oxygen species, than wild-type Arabidopsis (Jin et al., 2014). Many of these defects become milder under normal light. Therefore, it was proposed that HHL1 is involved in the repair and reassembly cycle of photodamaged PSII. Consistent with this hypothesis, HHL1 was found in both grana stacks and stroma lamellae, and PSII core subunits in thylakoid membranes isolated from HHL1-deficient plants were found to be less stable than those isolated from wild-type plants (Jin et al., 2014). Jin et al. (2014) also observed in vivo and in vitro interaction between HHL1 and LQY1, another protein involved in PSII repair. Because the majority of HHL1 is associated with PSII core monomers, it is likely that HHL1 "collaborates" with LQY1 and assists the reassembly of PSII core monomers and PSII-LHCII supercomplexes during PSII repair (Figure 2). In line with this hypothesis, the hhl1 lqy1 double mutant is more sensitive to high light than the single mutants (Jin et al., 2014).

MPH1 (Maintenance of PSII under High light 1) is a prolinerich intrinsic thylakoid membrane protein with a single-pass transmembrane domain; it is present in grana stacks, grana margins, and stroma lamellae (Liu and Last, 2015a,b). Under normal light, PSII activity and the composition of PSII complexes in MPH1-deficient mutants are similar to those in wild-type Arabidopsis. Under high light, the mph1 mutants have lower efficiency of PSII photochemistry, and lower amounts of PSIILHCII supercomplexes, PSII dimers, and PSII core monomers, than the wild type. Therefore, it was proposed that MPH1 has a role in protection and/or stabilization of PSII under high light (Liu and Last, 2015a,b). Consistent with this hypothesis, MPH1 was found to change its association under different light intensities: under normal light, the majority of MPH1 is associated with PSII core monomers; under high light, the majority of MPH1 becomes associated with PSII-LHCII supercomplexes (Liu and Last, 2015a). Because of the interactions between MPH1 and different PSII complexes under different light irradiance and the reductions in the amounts of PSII monomers and higher order PSII complexes, it is likely that MPH1 is involved in the assembly and/or stability of PSII core monomers and higher order PSII complexes under high light (Figure 2).

\section{CONCLUDING REMARKS}

Photosynthesis directly or indirectly provides chemical energy for nearly all life forms on earth. Due to the importance of photosynthesis, the structure, biogenesis, and maintenance of the photosynthetic apparatus have long been one of the major focuses of research. The combination of proteomics, Xray crystallography, and single-particle electron cryo-microscopy approaches has led to a comprehensive understanding of the structure and subunit composition of PSII. In addition, significant progresses have been made in the identification and functional studies of protein factors that are involved in de novo assembly and/or the repair and reassembly cycle of PSII. The inclusion of thylakoid protein trafficking/targeting systems and enzymes that catalyze important enzymatic steps, along with various assembly/stability factors allows a more comprehensive view of recent advances in this field. However, additional efforts are in great need to (1) dissect the precise functions of understudied assembly/stability factors or enzymes; and (2) build a protein interactome network that would provide a systems view of the interplay among different assembly/stability factors, enzymes, thylakoid protein trafficking/targeting systems, PSII assembly and repair complexes, and PSII subunits.

\section{AUTHOR CONTRIBUTIONS}

This review was written entirely by YL.

\section{ACKNOWLEDGMENTS}

This work was supported by the US National Science Foundation Grant MCB-1244008. 


\section{REFERENCES}

Adamska, I., and Kloppstech, K. (1991). Evidence for an association of the early light-inducible protein (ELIP) of pea with Photosystem II. Plant Mol. Biol. 16, 209-223. doi: 10.1007/BF00020553

Albiniak, A. M., Baglieri, J., and Robinson, C. (2012). Targeting of lumenal proteins across the thylakoid membrane. J. Exp. Bot. 63, 1689-1698. doi: 10.1093/jxb/ err444

Albrecht, V., Ingenfeld, A., and Apel, K. (2008). Snowy cotyledon 2: the identification of a zinc finger domain protein essential for chloroplast development in cotyledons but not in true leaves. Plant Mol. Biol. 66, 599-608. doi: 10.1007/s11103-008-9291-y

Anbudurai, P. R., Mor, T. S., Ohad, I., Shestakov, S. V., and Pakrasi, H. B. (1994). The $\operatorname{ctp} A$ gene encodes the $\mathrm{C}$-terminal processing protease for the $\mathrm{D} 1$ protein of the Photosystem II reaction center complex. Proc. Natl. Acad. Sci. U.S.A. 91, 8082-8086. doi: 10.1073/pnas.91.17.8082

Andersson, U., Heddad, M., and Adamska, I. (2003). Light stress-induced one-helix protein of the chlorophyll $a / b$-binding family associated with Photosystem I. Plant Physiol. 132, 811-820. doi: 10.1104/pp.102.019281

Armbruster, U., Zühlke, J., Rengstl, B., Kreller, R., Makarenko, E., Ruhle, T., et al. (2010). The Arabidopsis thylakoid protein PAM68 is required for efficient D1 biogenesis and Photosystem II assembly. Plant Cell 22, 3439-3460. doi: 10.1105/tpc.110.077453

Aro, E.-M., Suorsa, M., Rokka, A., Allahverdiyeva, Y., Paakkarinen, V., Saleem, A., et al. (2005). Dynamics of Photosystem II: a proteomic approach to thylakoid protein complexes. J. Exp. Bot. 56, 347-356. doi: 10.1093/jxb/eri041

Bailey, S., Thompson, E., Nixon, P. J., Horton, P., Mullineaux, C. W., Robinson, C., et al. (2002). A critical role for the Var2 FtsH homologue of Arabidopsis thaliana in the Photosystem II repair cycle in vivo. J. Biol. Chem. 277, 2006-2011. doi: 10.1074/jbc.M105878200

Bellafiore, S., Barneche, F., Peltier, G., and Rochaix, J. D. (2005). State transitions and light adaptation require chloroplast thylakoid protein kinase STN7. Nature 433, 892-895. doi: 10.1038/nature03286

Bhuiyan, N. H., Friso, G., Poliakov, A., Ponnala, L., and van Wijk, K. J. (2015). MET1 is a thylakoid-associated TPR protein involved in Photosystem II supercomplex formation and repair in Arabidopsis. Plant Cell 27, 262-285. doi: 10.1105/tpc.114.132787

Boehm, M., Yu, J., Krynicka, V., Barker, M., Tichy, M., Komenda, J., et al. (2012b). Subunit organization of a Synechocystis hetero-oligomeric thylakoid FtsH complex involved in Photosystem II repair. Plant Cell 24, 3669-3683. doi: 10.1105/tpc.112.100891

Boehm, M., Yu, J., Reisinger, V., Beckova, M., Eichacker, L. A., Schlodder, E., et al. (2012a). Subunit composition of CP43-less Photosystem II complexes of Synechocystis sp. PCC 6803: implications for the assembly and repair of Photosystem II. Phil. Trans. R. Soc. B 367, 3444-3454. doi: 10.1098/rstb.2012.0066

Bohne, A.-V., Schwarz, C., Schottkowski, M., Lidschreiber, M., Piotrowski, M., Zerges, W., et al. (2013). Reciprocal regulation of protein synthesis and carbon metabolism for thylakoid membrane biogenesis. PLoS Biol. 11:e1001482. doi: 10.1371/journal.pbio.1001482

Bonardi, V., Pesaresi, P., Becker, T., Schleiff, E., Wagner, R., Pfannschmidt, T., et al. (2005). Photosystem II core phosphorylation and photosynthetic acclimation require two different protein kinases. Nature 437, 1179-1182. doi: 10.1038 /nature 04016

Bricker, T., Roose, J. L., Zhang, P., and Frankel, L. (2013). The PsbP family of proteins. Photosynth. Res. 116, 235-250. doi: 10.1007/s11120-013-9820-7

Bricker, T. M., Roose, J. L., Fagerlund, R. D., Frankel, L. K., and Eaton-Rye, J. J. (2012). The extrinsic proteins of Photosystem II. Biochim. Biophys. Acta 1817, 121-142. doi: 10.1016/j.bbabio.2011.07.006

Cai, W., Ma, J., Chi, W., Zou, M., Guo, J., Lu, C., et al. (2010). Cooperation of LPA3 and LPA2 is essential for Photosystem II assembly in Arabidopsis. Plant Physiol. 154, 109-120. doi: 10.1104/pp.110.159558

Cai, W., Ma, J., Guo, J., and Zhang, L. (2008). Function of ROC4 in the efficient repair of photodamaged Photosystem II in Arabidopsis. Photochem. Photobiol. 84, 1343-1348. doi: 10.1111/j.1751-1097.2008.00448.x

Cain, P., Hall, M., Schröder, W. P., Kieselbach, T., and Robinson, C. (2009). A novel extended family of stromal thioredoxins. Plant Mol. Biol. 70, 273-281. doi: 10.1007/s11103-009-9471-4
Calderon, R. H., García-Cerdán, J. G., Malnoë, A., Cook, R., Russell, J. J. Gaw, C., et al. (2013). A Conserved rubredoxin is necessary for Photosystem II accumulation in diverse oxygenic photoautotrophs. J. Biol. Chem. 288, 26688-26696. doi: 10.1074/jbc.M113.487629

Casazza, A. P., Rossini, S., Rosso, M. G., and Soave, C. (2005). Mutational and expression analysis of ELIP1 and ELIP2 in Arabidopsis thaliana. Plant Mol. Biol. 58, 41-51. doi: 10.1007/s11103-005-4090-1

Charuvi, D., Kiss, V., Nevo, R., Shimoni, E., Adam, Z., and Reich, Z. (2012). Gain and loss of photosynthetic membranes during plastid differentiation in the shoot apex of Arabidopsis. Plant Cell 24, 1143-1157. doi: 10.1105/tpc.111.094458

Chassin, Y., Kapri-Pardes, E., Sinvany, G., Arad, T., and Adam, Z. (2002). Expression and characterization of the thylakoid lumen protease DegP1 from Arabidopsis. Plant Physiol. 130, 857-864. doi: 10.1104/pp.007922

Che, Y., Fu, A., Hou, X., McDonald, K., Buchanan, B. B., Huang, W., et al. (2013). $\mathrm{C}$-terminal processing of reaction center protein D1 is essential for the function and assembly of Photosystem II in Arabidopsis. Proc. Natl. Acad. Sci. U.S.A. 110, 16247-16252. doi: 10.1073/pnas.1313894110

Chen, H., Zhang, D., Guo, J., Wu, H., Jin, M., Lu, Q., et al. (2006a). A Psb27 homologue in Arabidopsis thaliana is required for efficient repair of photodamaged Photosystem II. Plant Mol. Biol. 61, 567-575. doi: 10.1007/s11103-006-0031-x

Chen, J., Burke, J. J., Velten, J., and Xin, Z. (2006b). FtsH11 protease plays a critical role in Arabidopsis thermotolerance. Plant J. 48, 73-84. doi: $10.1111 / \mathrm{j} .1365$ 313X.2006.02855.x

Chen, M., Choi, Y., Voytas, D. F., and Rodermel, S. (2000). Mutations in the Arabidopsis VAR2 locus cause leaf variegation due to the loss of a chloroplast FtsH protease. Plant J. 22, 303-313. doi: 10.1046/j.1365-313x.2000.00738.x

Chidgey, J. W., Linhartová, M., Komenda, J., Jackson, P. J., Dickman, M. J., Canniffe, D. P., et al. (2014). A cyanobacterial chlorophyll synthase-HliD complex associates with the Ycf39 protein and the YidC/Alb3 insertase. Plant Cell 26, 1267-1279. doi: 10.1105/tpc.114.124495

Cline, K., and Dabney-Smith, C. (2008). Plastid protein import and sorting: different paths to the same compartments. Curr. Opin. Plant Biol. 11, 585-592. doi: 10.1016/j.pbi.2008.10.008

Cline, K., and Theg, S. (2007). "The Sec and Tat protein translocation pathways in chloroplasts," in The Enzymes: Molecular Machines Involved in Protein Transport across Cellular Membranes, eds R. E. Dalbey, C. M. Koehler, and F. Tamanoi (Waltham, MA: Academic Press), 463-492.

da Fonseca, P., Morris, E. P., Hankamer, B., and Barber, J. (2002). Electron crystallographic study of Photosystem II of the cyanobacterium Synechococcus elongatus. Biochemistry 41, 5163-5167. doi: 10.1021/bi0120650

Dall'Osto, L., Bressan, M., and Bassi, R. (2015). Biogenesis of light harvesting proteins. Biochim. Biophys. Acta 1847, 861-871. doi: 10.1016/j.bbabio.2015.02.009

Dietzel, L., Bräutigam, K., Steiner, S., Schüffler, K., Lepetit, B., Grimm, B., et al. (2011). Photosystem II supercomplex remodeling serves as an entry mechanism for state transitions in Arabidopsis. Plant Cell 23, 2964-2977. doi: 10.1105/tpc.111.087049

Dobáková, M., Sobotka, R., Tichy, M., and Komenda, J. (2009). Psb28 protein is involved in the biogenesis of the Photosystem II inner antenna CP47 (PsbB) in the cyanobacterium Synechocystis sp. PCC 6803. Plant Physiol. 149, 1076-1086. doi: 10.1104/pp.108.130039

Dominguez-Solis, J. R., He, Z. Y., Lima, A., Ting, J., Buchanan, B. B., and Luan, S. (2008). A cyclophilin links redox and light signals to cysteine biosynthesis and stress responses in chloroplasts. Proc. Natl. Acad. Sci. U.S.A. 105, 16386-16391. doi: $10.1073 /$ pnas.0808204105

Feige, M. J., and Hendershot, L. M. (2011). Disulfide bonds in ER protein folding and homeostasis. Curr. Opin. Cell Biol. 23, 167-175. doi: 10.1016/j.ceb.2010.10.012

Feng, W.-K., Wang, L., Lu, Y., and Wang, X.-Y. (2011). A protein oxidase catalysing disulfide bond formation is localized to the chloroplast thylakoids. FEBS J. 278, 3419-3430. doi: 10.1111/j.1742-4658.2011.08265.x

Fischer, G., Tradler, T., and Zarnt, T. (1998). The mode of action of peptidyl prolyl cis/trans isomerases in vivo: binding vs. catalysis. FEBS Lett. 426, 17-20. doi: 10.1016/S0014-5793(98)00242-7

Fristedt, R., Herdean, A., Blaby-Haas, C. E., Mamedov, F., Merchant, S. S., Last, R. L., et al. (2015). PHOTOSYSTEM II PROTEIN33, a protein conserved 
in the plastid lineage, is associated with the chloroplast thylakoid membrane and provides stability to Photosystem II supercomplexes in Arabidopsis. Plant Physiol. 167, 481-492. doi: 10.1104/pp.114.253336

Fu, A., He, Z. Y., Cho, H. S., Lima, A., Buchanan, B. B., and Luan, S. (2007). A chloroplast cyclophilin functions in the assembly and maintenance of Photosystem II in Arabidopsis thaliana. Proc. Natl. Acad. Sci. U.S.A. 104, 15947-15952. doi: 10.1073/pnas.0707851104

Fulgosi, H., Vener, A. V., Altschmied, L., Herrmann, R. G., and Andersson, B. (1998). A novel multi-functional chloroplast protein: identification of a 40 $\mathrm{kDa}$ immunophilin-like protein located in the thylakoid lumen. EMBO J. 17, 1577-1587. doi: 10.1093/emboj/17.6.1577

Göhre, V., Ossenbühl, F., Crèvecoeur, M., Eichacker, L. A., and Rochaix, J.D. (2006). One of two Alb3 proteins is essential for the assembly of the photosystems and for cell survival in Chlamydomonas. Plant Cell 18, 1454-1466. doi: 10.1105/tpc.105.038695

Goral, T. K., Johnson, M. P., Brain, A. P., Kirchhoff, H., Ruban, A. V., and Mullineaux, C. W. (2010). Visualizing the mobility and distribution of chlorophyll proteins in higher plant thylakoid membranes: effects of photoinhibition and protein phosphorylation. Plant J. 62, 948-959. doi: 10.1111/j.1365-313x.2010.04207.x

Haubühl, K., Andersson, B., and Adamska, I. (2001). A chloroplast DegP2 protease performs the primary cleavage of the photodamaged D1 protein in plant Photosystem II. EMBO J. 20, 713-722. doi: 10.1093/emboj/20.4.713

He, Z., Li, L., and Luan, S. (2004). Immunophilins and parvulins. Superfamily of peptidyl prolyl isomerases in Arabidopsis. Plant Physiol. 134, 1248-1267. doi: 10.1104/pp.103.031005

Heddad, M., Engelken, J., and Adamska, I. (2012). "Light stress proteins in viruses, cyanobacteria and photosynthetic eukaryota," in Photosynthesis, eds J. J. Eaton-Rye, B. C. Tripathy, and T. D. Sharkey (Dordrecht: Springer), 299-317.

Heddad, M., Norén, H., Reiser, V., Dunaeva, M., Andersson, B., and Adamska, I. (2006). Differential expression and localization of early light-induced proteins in Arabidopsis. Plant Physiol. 142, 75-87. doi: 10.1104/pp.106.081489

Henry, R., Goforth, R., and Schunemann, D. (2007). "Chloroplast SRP/FtsY and Alb3 in protein integration into the thylakoid membrane," in The Enzymes: Molecular Machines Involved in Protein Transport across Cellular Membranes, eds R. E. Dalbey, C. M. Koehler, and F. Tamanoi (Waltham, MA: Academic Press), 493-521.

Hentze, M. W. (1994). Enzymes as RNA-binding proteins: a role for (di)nucleotidebinding domains? Trends Biochem. Sci. 19, 101-103. doi: 10.1016/09680004(94)90198-8

Herbstová, M., Tietz, S., Kinzel, C., Turkina, M. V., and Kirchhoff, H. (2012). Architectural switch in plant photosynthetic membranes induced by light stress. Proc. Natl. Acad. Sci. U.S.A. 109, 20130-20135. doi: 10.1073/pnas. 1214265109

Hoober, J. K., Boyd, C. O., and Paavola, L. G. (1991). Origin of thylakoid membranes in Chlamydomonas reinhardtii $y-1$ at $38^{\circ} \mathrm{C}$. Plant Physiol. 96, 1321-1328. doi: 10.1104/pp.96.4.1321

Hoober, J. K., Eggink, L. L., and Chen, M. (2007). Chlorophylls, ligands and assembly of light-harvesting complexes in chloroplasts. Photosynth. Res. 94, 387-400. doi: 10.1007/s11120-007-9181-1

Houston, N. L., Fan, C., Xiang, Q.-Y., Schulze, J.-M., Jung, R., and Boston, R. S. (2005). Phylogenetic analyses identify 10 classes of the protein disulfide isomerase family in plants, including single-domain protein disulfide isomerase-related proteins. Plant Physiol. 137, 762-778. doi: 10.1104/pp.104.056507

Huang, J., Taylor, J. P., Chen, J. G., Uhrig, J. F., Schnell, D. J., Nakagawa, T., et al. (2006). The plastid protein THYLAKOID FORMATION1 and the plasma membrane G-protein GPA1 interact in a novel sugar-signaling mechanism in Arabidopsis. Plant Cell 18, 1226-1238. doi: 10.1105/tpc.105.037259

Huang, W., Chen, Q., Zhu, Y., Hu, F., Zhang, L., Ma, Z., et al. (2013). Arabidopsis thylakoid formation 1 is a critical regulator for dynamics of PSII-LHCII complexes in leaf senescence and excess light. Mol. Plant 6, 1673-1691. doi: $10.1093 / \mathrm{mp} / \mathrm{sst} 069$

Huesgen, P. F., Schuhmann, H., and Adamska, I. (2005). The family of Deg proteases in cyanobacteria and chloroplasts of higher plants. Physiol. Plant. 123, 413-420. doi: 10.1111/j.1399-3054.2005.00458.x

Huesgen, P. F., Schuhmann, H., and Adamska, I. (2006). Photodamaged D1 protein is degraded in Arabidopsis mutants lacking the Deg2 protease. FEBS Lett. 580, 6929-6932. doi: 10.1016/j.febslet.2006 11.058

Huesgen, P. F., Schuhmann, H., and Adamska, I. (2009). Deg/HtrA proteases as components of a network for Photosystem II quality control in chloroplasts and cyanobacteria. Res. Microbiol. 160, 726-732. doi: 10.1016/j.resmic.2009.08.005

Hutin, C., Nussaume, L., Moise, N., Moya, I., Kloppstech, K., and Havaux, M. (2003). Early light-induced proteins protect Arabidopsis from photooxidative stress. Proc. Natl. Acad. Sci. U.S.A. 100, 4921-4926. doi: 10.1073/pnas.0736939100

Ifuku, K. (2014). The PsbP and PsbQ family proteins in the photosynthetic machinery of chloroplasts. Plant Physiol. Biochem. 81, 108-114. doi: 10.1016/j.plaphy.2014.01.001

Ikeuchi, M., Koike, H., and Inoue, Y. (1989). N-terminal sequencing of lowmolecular-mass components in cyanobacterial Photosystem II core complex: two components correspond to unidentified open reading frames of plant chloroplast DNA. FEBS Lett. 253, 178-182. doi: 10.1016/0014-5793(89)80954-8

Ingelsson, B., Shapiguzov, A., Kieselbach, T., and Vener, A. V. (2009). Peptidyl prolyl isomerase activity in chloroplast thylakoid lumen is a dispensable function of immunophilins in Arabidopsis thaliana. Plant Cell Physiol. 50, 1801-1814. doi: 10.1093/pcp/pcp122

Ishihara, S., Takabayashi, A., Ido, K., Endo, T., Ifuku, K., and Sato, F. (2007). Distinct functions for the two PsbP-like proteins PPL1 and PPL2 in the chloroplast thylakoid lumen of Arabidopsis. Plant Physiol. 145, 668-679. doi: 10.1104/pp.107.105866

Ishikawa, A., Tanaka, H., Kato, C., Iwasaki, Y., and Asahi, T. (2005a). Molecular characterization of the ZKT gene encoding a protein with PDZ, K-Box, and TPR motifs in Arabidopsis. Biosci. Biotechnol. Biochem. 69, 972-978. doi: 10.1271/bbb.69.972

Ishikawa, Y., Schroder, W. P., and Funk, C. (2005b). Functional analysis of the PsbP-like protein (sll1418) in Synechocystis sp. PCC 6803. Photosynth. Res. 84, 257-262. doi: 10.1007/s11120-005-0477-8

Jackson, S. A., Hinds, M. G., and Eaton-Rye, J. J. (2012). Solution structure of CyanoP from Synechocystis sp. PCC 6803: new insights on the structural basis for functional specialization amongst PsbP family proteins. Biochim. Biophys. Acta 1817, 1331-1338. doi: 10.1016/j.bbabio.2012.02.032

Jansson, S. (1999). A guide to the Lhc genes and their relatives in Arabidopsis. Trends Plant Sci. 4, 236-240. doi: 10.1016/S1360-1385(99) 01419-3

Järvi, S., Gollan, P. J., and Aro, E.-M. (2013). Understanding the roles of the thylakoid lumen in photosynthesis regulation. Front. Plant Sci. 4:434. doi: 10.3389/fpls.2013.00434

Järvi, S., Suorsa, M., and Aro, E.-M. (2015). Photosystem II repair in plant chloroplasts-Regulation, assisting proteins and shared components with Photosystem II biogenesis. Biochim. Biophys. Acta 1847, 900-909. doi: 10.1016/j.bbabio.2015.01.006

Jessop, C. E., Chakravarthi, S., Garbi, N., Hämmerling, G. J., Lovell, S., and Bulleid, N. J. (2007). ERp57 is essential for efficient folding of glycoproteins sharing common structural domains. EMBO J. 26, 28-40. doi: 10.1038/sj.emboj.7601505

Jin, H., Liu, B., Luo, L., Feng, D., Wang, P., Liu, J., et al. (2014) HYPERSENSITIVE TO HIGH LIGHT1 interacts with LOW QUANTUM YIELD OF PHOTOSYSTEM II1 and functions in protection of Photosystem II from photodamage in Arabidopsis. Plant Cell 26, 1213-1229. doi: 10.1105/tpc.113.122424

Jung, K.-H., Lee, J., Dardick, C., Seo, Y.-S., Cao, P., Canlas, P., et al. (2008). Identification and functional analysis of light-responsive unique genes and gene family members in rice. PLoS Genet. 4:e1000164. doi: 10.1371/journal.pgen.1000164

Kapri-Pardes, E., Naveh, L., and Adam, Z. (2007). The thylakoid lumen protease Deg1 is involved in the repair of Photosystem II from photoinhibition in Arabidopsis. Plant Cell 19, 1039-1047. doi: 10.1105/tpc.106.046573

Karamoko, M., Cline, S., Redding, K., Ruiz, N., and Hamel, P. P. (2011). Lumen Thiol Oxidoreductase1, a disulfide bond-forming catalyst, is required for the assembly of Photosystem II in Arabidopsis. Plant Cell 23, 4462-4475. doi: 10.1105/tpc.111.089680

Karamoko, M., Gabilly, S., and Hamel, P. P. (2013). Operation of trans-thylakoid thiol-metabolizing pathways in photosynthesis. Front. Plant Sci. 4:476. doi: 10.3389/fpls.2013.00476 
Karim, S., Alezzawi, M., Garcia-Petit, C., Solymosi, K., Khan, N. Z., Lindquist, E., et al. (2014). A novel chloroplast localized Rab GTPase protein CPRabA5e is involved in stress, development, thylakoid biogenesis and vesicle transport in Arabidopsis. Plant Mol. Biol. 84, 675-692. doi: 10.1007/s11103-013-0161-x

Karim, S., and Aronsson, H. (2014). The puzzle of chloroplast vesicle transport involvement of GTPases. Front. Plant Sci. 5:472. doi: 10.3389/fpls.2014.00472

Kashino, Y., Lauber, W. M., Carroll, J. A., Wang, Q. J., Whitmarsh, J., Satoh, K., et al. (2002). Proteomic analysis of a highly active Photosystem II preparation from the cyanobacterium Synechocystis sp. PCC 6803 reveals the presence of novel polypeptides. Biochemistry 41, 8004-8012. doi: 10.1021/bi026012+

Kato, Y., Miura, E., Ido, K., Ifuku, K., and Sakamoto, W. (2009). The variegated mutants lacking chloroplastic FtsHs are defective in D1 degradation and accumulate reactive oxygen species. Plant Physiol. 151, 1790-1801. doi: 10.1104/pp.109.146589

Kato, Y., Miura, E., Matsushima, R., and Sakamoto, W. (2007). White leaf sectors in yellow variegated 2 are formed by viable cells with undifferentiated plastids. Plant Physiol. 144, 952-960. doi: 10.1104/pp.107.099002

Kato, Y., Sun, X., Zhang, L., and Sakamoto, W. (2012). Cooperative D1 degradation in the Photosystem II repair mediated by chloroplastic proteases in Arabidopsis. Plant Physiol. 159, 1428-1439. doi: 10.1104/pp.112.199042

Keren, N., Ohkawa, H., Welsh, E. A., Liberton, M., and Pakrasi, H. B. (2005). Psb29, a conserved 22-kD protein, functions in the biogenesis of Photosystem II complexes in Synechocystis and Arabidopsis. Plant Cell 17, 2768-2781. doi: 10.1105/tpc.105.035048

Khan, N. Z., Lindquist, E., and Aronsson, H. (2013). New putative chloroplast vesicle transport components and cargo proteins revealed using a bioinformatics approach: an Arabidopsis model. PLoS ONE 8:e59898. doi: 10.1371/journal.pone.0059898

Khatoon, M., Inagawa, K., Pospisil, P., Yamashita, A., Yoshioka, M., Lundin, B., et al. (2009). Quality control of Photosystem II: Thylakoid unstacking is necessary to avoid further damage to the D1 protein and to facilitate D1 degradation under light stress in spinach thylakoids. J. Biol. Chem. 284, 25343-25352. doi: 10.1074/jbc.M109.007740

Klinkert, B., Ossenbuhl, F., Sikorski, M., Berry, S., Eichacker, L., and Nickelsen, J. (2004). PratA, a periplasmic tetratricopeptide repeat protein involved in biogenesis of Photosystem II in Synechocystis sp. PCC 6803. J. Biol. Chem. 279, 44639-44644. doi: 10.1074/jbc.M405393200

Knoppová, J., Sobotka, R., Tich, M., Yu, J., Konik, P., Halada, P., et al. (2014). Discovery of a chlorophyll binding protein complex involved in the early steps of Photosystem II assembly in Synechocystis. Plant Cell 26, 1200-1212. doi: $10.1105 /$ tpc. 114.123919

Komenda, J., Knoppová, J., Kopečná, J., Sobotka, R., Halada, P., Yu, J., et al. (2012b). The Psb27 assembly factor binds to the CP43 complex of Photosystem II in the cyanobacterium Synechocystis sp. PCC 6803. Plant Physiol. 158, 476-486. doi: 10.1104/pp.111.184184

Komenda, J., Nickelsen, J., Tich, M., Prášil, O., Eichacker, L. A., and Nixon, P. J. (2008). The cyanobacterial homologue of HCF136/YCF48 is a component of an early Photosystem II assembly complex and is important for both the efficient assembly and repair of Photosystem II in Synechocystis sp. PCC 6803. J. Biol. Chem. 283, 22390-22399. doi: 10.1074/jbc.M801917200

Komenda, J., Sobotka, R., and Nixon, P. J. (2012a). Assembling and maintaining the Photosystem II complex in chloroplasts and cyanobacteria. Curr. Opin. Plant Biol. 15, 245-251. doi: 10.1016/j.pbi.2012.01.017

Krech, K., Fu, H.-Y., Thiele, W., Ruf, S., Schöttler, M. A., and Bock, R. (2013). Reverse genetics in complex multigene operons by co-transformation of the plastid genome and its application to the open reading frame previously designated psbN. Plant J. 75, 1062-1074. doi: 10.1111/tpj.12256

Kroll, D., Meierhoff, K., Bechtold, N., Kinoshita, M., Westphal, S., Vothknecht, U. C., et al. (2001). VIPP1, a nuclear gene of Arabidopsis thaliana essential for thylakoid membrane formation. Proc. Natl. Acad. Sci. U.S.A. 98, 4238-4242. doi: 10.1073/pnas.061500998

Krzywda, S., Brzozowski, A. M., Verma, C., Karata, K., Ogura, T., and Wilkinson, A. J. (2002). The crystal structure of the AAA domain of the ATP-dependent protease FtsH of Escherichia coli at $1.5 \AA$ A resolution. Structure 10, 1073-1083. doi: 10.1016/S0969-2126(02)00806-7

Kwon, K.-C., and Cho, M. H. (2008). Deletion of the chloroplast-localized AtTerC gene product in Arabidopsis thaliana leads to loss of the thylakoid membrane and to seedling lethality. Plant J. 55, 428-442. doi: 10.1111/j.1365313X.2008.03523.x

Liao, D. I., Qian, J., Chisholm, D. A., Jordan, D. B., and Diner, B. A. (2000). Crystal structures of the Photosystem II D1 C-terminal processing protease. Nat. Struct. Biol. 7, 749-753. doi: 10.1038/78973

Lima, A., Lima, S., Wong, J. H., Phillips, R. S., Buchanan, B. B., and Luan, S. (2006). A redox-active FKBP-type immunophilin functions in accumulation of the Photosystem II supercomplex in Arabidopsis thaliana. Proc. Natl. Acad. Sci. U.S.A. 103, 12631-12636. doi: 10.1073/pnas.0605452103

Lindahl, M., Spetea, C., Hundal, T., Oppenheim, A. B., Adam, Z., and Andersson, B. (2000). The thylakoid FtsH protease plays a role in the light-induced turnover of the Photosystem II D1 protein. Plant Cell 12, 419-432. doi: 10.1105/tpc.12.3.419

Lindahl, M., Tabak, S., Cseke, L., Pichersky, E., Andersson, B., and Adam, Z. (1996). Identification, characterization, and molecular cloning of a homologue of the bacterial FtsH protease in chloroplasts of higher plants. J. Biol. Chem. 271, 29329-29334. doi: 10.1074/jbc.271.46.29329

Link, S., Engelmann, K., Meierhoff, K., and Westhoff, P. (2012). The atypical short-chain dehydrogenases HCF173 and HCF244 are jointly involved in translational initiation of the $p s b A$ mRNA of Arabidopsis. Plant Physiol. 160, 2202-2218. doi: 10.1104/pp.112.205104

Lippuner, V., Chou, I. T., Scott, S. V., Ettinger, W. F., Theg, S. M., and Gasser, C. S. (1994). Cloning and characterization of chloroplast and cytosolic forms of cyclophilin from Arabidopsis thaliana. J. Biol. Chem. 269, 7863-7868.

Liu, H., Huang, R. Y.-C., Chen, J., Gross, M. L., and Pakrasi, H. B. (2011b). Psb27, a transiently associated protein, binds to the chlorophyll binding protein CP43 in Photosystem II assembly intermediates. Proc. Natl. Acad. Sci. U.S.A. 108, 18536-18541. doi: 10.1073/pnas.1111597108

Liu, H., Roose, J. L., Cameron, J. C., and Pakrasi, H. B. (2011a). A genetically tagged Psb27 protein allows purification of two consecutive Photosystem II (PSII) assembly intermediates in Synechocystis 6803, a cyanobacterium. J. Biol. Chem. 286, 24865-24871. doi: 10.1074/jbc.M111.246231

Liu, J., and Last, R. L. (2015a). A land plant-specific thylakoid membrane protein contributes to Photosystem II maintenance in Arabidopsis thaliana. Plant J. 82, 731-743. doi: 10.1111/tpj.12845

Liu, J., and Last, R. L. (2015b). MPH1 is a thylakoid membrane protein involved in protecting Photosystem II from photodamage in land plants. Plant Signal. Behav. 10:e1076602. doi: 10.1080/15592324.2015.1076602

Liu, L.-N., Chen, X.-L., Zhang, Y.-Z., and Zhou, B.-C. (2005). Characterization, structure and function of linker polypeptides in phycobilisomes of cyanobacteria and red algae: an overview. Biochim. Biophys. Acta 1708, 133-142. doi: 10.1016/j.bbabio.2005.04.001

Liu, Z., Yan, H., Wang, K., Kuang, T., Zhang, J., Gui, L., et al. (2004). Crystal structure of spinach major light-harvesting complex at $2.72 \AA$ resolution. Nature 428, 287-292. doi: 10.1038/nature02373

Lohscheider, J. N., Rojas-Stutz, M. C., Rothbart, M., Andersson, U., Funck, D., Mendgen, K., et al. (2015). Altered levels of LIL3 isoforms in Arabidopsis lead to disturbed pigment-protein assembly and chlorophyll synthesis, chlorotic phenotype and impaired photosynthetic performance. Plant Cell Environ. 38, 2115-2127. doi: $10.1111 /$ pce. 12540

Lu, Y. (2011). The occurrence of a thylakoid-localized small zinc finger protein in land plants. Plant Signal. Behav. 6, 1181-1185. doi: 10.4161/psb.6.12.18022

Lu, Y., Hall, D. A., and Last, R. L. (2011). A small zinc finger thylakoid protein plays a role in maintenance of Photosystem II in Arabidopsis thaliana. Plant Cell 23, 1861-1875. doi: 10.1105/tpc.111.085456

Lu, Y., Wang, H.-R., Li, H., Cui, H.-R., Feng, Y.-G., and Wang, X.-Y. (2013). A chloroplast membrane protein LTO1/AtVKOR involving in redox regulation and ROS homeostasis. Plant Cell Rep. 32, 1427-1440. doi: 10.1007/s00299-0131455-9

Luciński, R., Misztal, L., Samardakiewicz, S., and Jackowski, G. (2011a). Involvement of Deg5 protease in wounding-related disposal of PsbF apoprotein. Plant Physiol. Biochem. 49, 311-320. doi: 10.1016/j.plaphy.2011.01.001

Luciński, R., Misztal, L., Samardakiewicz, S., and Jackowski, G. (2011b). The thylakoid protease Deg2 is involved in stress-related degradation of the Photosystem II light-harvesting protein Lhcb6 in Arabidopsis thaliana. New Phytol. 192, 74-86. doi: 10.1111/j.1469-8137.2011.03782.x 
Ma, J., Peng, L., Guo, J., Lu, Q., Lu, C., and Zhang, L. (2007). LPA2 is required for efficient assembly of Photosystem II in Arabidopsis thaliana. Plant Cell 19, 1980-1993. doi: 10.1105/tpc.107.050526

Mabbitt, P. D., Wilbanks, S. M., and Eaton-Rye, J. J. (2014). Structure and function of the hydrophilic Photosystem II assembly proteins: Psb27, Psb28 and Ycf48. Plant Physiol. Biochem. 81, 96-107. doi: 10.1016/j.plaphy.2014.02.013

Mann, N. H., Novac, N., Mullineaux, C. W., Newman, J., Bailey, S., and Robinson, C. (2000). Involvement of an FtsH homologue in the assembly of functional Photosystem I in the cyanobacterium Synechocystis sp. PCC 6803. FEBS Lett. 479, 72-77. doi: 10.1016/S0014-5793(00)01871-8

Meurer, J., Plücken, H., Kowallik, K. V., and Westhoff, P. (1998). A nuclearencoded protein of prokaryotic origin is essential for the stability of Photosystem II in Arabidopsis thaliana. EMBO J. 17, 5286-5297. doi: 10.1093/emboj/17.18.5286

Michoux, F., Boehm, M., Bialek, W., Takasaka, K., Maghlaoui, K., Barber, J., et al. (2014). Crystal structure of CyanoQ from the thermophilic cyanobacterium Thermosynechococcus elongatus and detection in isolated Photosystem II complexes. Photosynth. Res. 122, 57-67. doi: 10.1007/s11120-014-0010-z

Michoux, F., Takasaka, K., Boehm, M., Nixon, P. J., and Murray, J. W. (2010). Structure of CyanoP at $2.8 \AA$ : implications for the evolution and function of the PsbP subunit of Photosystem II. Biochemistry 49, 7411-7413. doi: $10.1021 /$ bi1011145

Mork-Jansson, A. E., Gargano, D., Kmiec, K., Furnes, C., Shevela, D., and Eichacker, L. A. (2015). Lil3 dimerization and chlorophyll binding in Arabidopsis thaliana. FEBS Lett. 589, 3064-3070. doi: 10.1016/j.febslet.2015.08.023

Morré, D. J., Selldén, G., Sundqvist, C., and Sandelius, A. S. (1991). Stromal low temperature compartment derived from the inner membrane of the chloroplast envelope. Plant Physiol. 97, 1558-1564. doi: 10.1104/pp.97.4.1558

Mulo, P., Sirpiö, S., Suorsa, M., and Aro, E. M. (2008). Auxiliary proteins involved in the assembly and sustenance of Photosystem II. Photosynth. Res. 98, 489-501. doi: 10.1007/s11120-008-9320-3

Muranaka, A., Watanabe, S., Sakamoto, A., and Shimada, H. (2012). Arabidopsis cotyledon chloroplast biogenesis factor CYO1 uses glutathione as an electron donor and interacts with PSI (A1 and A2) and PSII (CP43 and CP47) subunits. J. Plant Physiol. 169, 1212-1215. doi: 10.1016/j.jplph.2012.04.001

Nagy, E., Henics, T., Eckert, M., Miseta, A., Lightowlers, R. N., and Kellermayer, M. (2000). Identification of the NAD(+)-binding fold of glyceraldehyde-3phosphate dehydrogenase as a novel RNA-binding domain. Biochem. Biophys. Res. Commun. 275, 253-260. doi: 10.1006/bbrc.2000.3246

Nath, K., Poudyal, R. S., Eom, J.-S., Park, Y. S., Zulfugarov, I. S., Mishra, S. R., et al. (2013). Loss-of-function of OsSTN8 suppresses the Photosystem II core protein phosphorylation and interferes with the Photosystem II repair mechanism in rice (Oryza sativa). Plant J. 76, 675-686. doi: 10.1111/tpj.12331

Nickelsen, J., and Rengstl, B. (2013). Photosystem II assembly: from cyanobacteria to plants. Annu. Rev. Plant Biol. 64, 609-635. doi: 10.1146/annurev-arplant050312-120124

Nickelsen, J., and Zerges, W. (2013). Thylakoid biogenesis has joined the new era of bacterial cell biology. Front. Plant Sci. 4:458. doi: 10.3389/fpls.2013. 00458

Nield, J., and Barber, J. (2006). Refinement of the structural model for the Photosystem II supercomplex of higher plants. Biochim. Biophys. Acta 1757, 353-361. doi: 10.1016/j.bbabio.2006.03.019

Niwa, H., Tsuchiya, D., Makyio, H., Yoshida, M., and Morikawa, K. (2002). Hexameric ring structure of the ATPase domain of the membraneintegrated metalloprotease FtsH from Thermus thermophilus HB8. Structure 10, 1415-1423. doi: 10.1016/S0969-2126(02)00855-9

Nixon, P. J., Barker, M., Boehm, M., de Vries, R., and Komenda, J. (2005). FtsHmediated repair of the Photosystem II complex in response to light stress. J. Exp. Bot. 56, 357-363. doi: 10.1093/jxb/eri021

Nixon, P. J., Michoux, F., Yu, J., Boehm, M., and Komenda, J. (2010). Recent advances in understanding the assembly and repair of Photosystem II. Ann. Bot. 106, 1-16. doi: 10.1093/aob/mcq059

Nixon, P. J., Trost, J. T., and Diner, B. A. (1992). Role of the carboxy terminus of polypeptide D1 in the assembly of a functional water-oxidizing manganese cluster in Photosystem II of the cyanobacterium Synechocystis sp. PCC 6803: assembly requires a free carboxyl group at C-terminal position 344 . Biochemistry 31, 10859-10871. doi: 10.1021/bi00159a029
Nowaczyk, M. M., Hebeler, R., Schlodder, E., Meyer, H. E., Warscheid, B., and Rögner, M. (2006). Psb27, a cyanobacterial lipoprotein, is involved in the repair cycle of Photosystem II. Plant Cell 18, 3121-3131. doi: 10.1105/tpc.106.042671

Ossenbühl, F., Göhre, V., Meurer, J., Krieger-Liszkay, A., Rochaix, J.-D., and Eichacker, L. A. (2004). Efficient assembly of Photosystem II in Chlamydomonas reinhardtii requires Alb3.1p, a homolog of Arabidopsis ALBINO3. Plant Cell 16, 1790-1800. doi: 10.1105/tpc.023226

Park, S. W., Li, W., Viehhauser, A., He, B., Kim, S., Nilsson, A. K., et al. (2013). Cyclophilin 20-3 relays a 12-oxo-phytodienoic acid signal during stress responsive regulation of cellular redox homeostasis. Proc. Natl. Acad. Sci. U.S.A. 110, 9559-9564. doi: 10.1073/pnas.1218872110

Pasch, J. C., Nickelsen, J., and Schünemann, D. (2005). The yeast split-ubiquitin system to study chloroplast membrane protein interactions. Appl. Microbiol. Biotechnol. 69, 440-447. doi: 10.1007/s00253-005-0029-3

Peng, L. W., Ma, J. F., Chi, W., Guo, J. K., Zhu, S. Y., Lu, Q. T., et al. (2006). LOW PSII ACCUMULATION1 is involved in efficient assembly of Photosystem II in Arabidopsis thaliana. Plant Cell 18, 955-969. doi: 10.1105/tpc.105.037689

Persson, B., Kallberg, Y., Bray, J. E., Bruford, E., Dellaporta, S. L., Favia, A. D., et al. (2009). The SDR (short-chain dehydrogenase/reductase and related enzymes) nomenclature initiative. Chem. Biol. Interact. 178, 94-98. doi: 10.1016/j.cbi.2008.10.040

Pesaresi, P., Pribil, M., Wunder, T., and Leister, D. (2011). Dynamics of reversible protein phosphorylation in thylakoids of flowering plants: the roles of STN7, STN8 and TAP38. Biochim. Biophys. Acta 1807, 887-896. doi: 10.1016/j.bbabio.2010.08.002

Pioli, P. A., Hamilton, B. J., Connolly, J. E., Brewer, G., and Rigby, W. F. (2002). Lactate dehydrogenase is an AU-rich element-binding protein that directly interacts with AUF1. J. Biol. Chem. 277, 35738-35745. doi: 10.1074/jbc.M204002200

Plücken, H., Müller, B., Grohmann, D., Westhoff, P., and Eichacker, L. A. (2002). The HCF136 protein is essential for assembly of the Photosystem II reaction center in Arabidopsis thaliana. FEBS Lett. 532, 85-90. doi: 10.1016/S00145793(02)03634-7

Pribil, M., Pesaresi, P., Hertle, A., Barbato, R., and Leister, D. (2010). Role of plastid protein phosphatase TAP38 in LHCII dephosphorylation and thylakoid electron flow. PLoS Biol. 8:e1000288. doi: 10.1371/journal.pbio.1000288

Rast, A., Heinz, S., and Nickelsen, J. (2015). Biogenesis of thylakoid membranes. Biochim. Biophys. Acta 1847, 821-830. doi: 10.1016/j.bbabio.2015.01.007

Rintamaki, E., Salonen, M., Suoranta, U. M., Carlberg, I., Andersson, B., and Aro, E. M. (1997). Phosphorylation of light-harvesting complex II and Photosystem II core proteins shows different irradiance-dependent regulation in vivo: application of phosphothreonine antibodies to analysis of thylakoid phosphoproteins. J. Biol. Chem. 272, 30476-30482. doi: $10.1074 /$ jbc. 272.48 .30476

Rokka, A., Aro, E.-M., Herrmann, R. G., Andersson, B., and Vener, A. V. (2000). Dephosphorylation of Photosystem II reaction center proteins in plant photosynthetic membranes as an immediate response to abrupt elevation of temperature. Plant Physiol. 123, 1525-1536. doi: 10.1104/pp.123.4.1525

Rokka, A., Suorsa, M., Saleem, A., Battchikova, N., and Aro, E. M. (2005). Synthesis and assembly of thylakoid protein complexes: multiple assembly steps of Photosystem II. Biochem. J. 388, 159-168. doi: 10.1042/BJ20042098

Romano, P. G., Horton, P., and Gray, J. E. (2004). The Arabidopsis cyclophilin gene family. Plant Physiol. 134, 1268-1282. doi: 10.1104/pp.103.022160

Rossini, S., Casazza, A. P., Engelmann, E. C. M., Havaux, M., Jennings, R. C., and Soave, C. (2006). Suppression of both ELIP1 and ELIP2 in Arabidopsis does not affect tolerance to photoinhibition and photooxidative stress. Plant Physiol. 141, 1264-1273. doi: 10.1104/pp.106.083055

Sakamoto, W., Tamura, T., Hanba-Tomita, Y., Sodmergen, and Murata, M. (2002). The VAR1 locus of Arabidopsis encodes a chloroplastic FtsH and is responsible for leaf variegation in the mutant alleles. Genes Cells 7, 769-780. doi: 10.1046/j.1365-2443.2002.00558.x

Sakamoto, W., Zaltsman, A., Adam, Z., and Takahashi, Y. (2003). Coordinated regulation and complex formation of YELLOW VARIEGATED1 and YELLOW VARIEGATED2, chloroplastic FtsH metalloproteases involved in the repair cycle of Photosystem II in Arabidopsis thylakoid membranes. Plant Cell 15, 2843-2855. doi: 10.1105/tpc.017319

Samol, I., Shapiguzov, A., Ingelsson, B., Fucile, G., Crèvecoeur, M., Vener, A. V., et al. (2012). Identification of a Photosystem II phosphatase 
involved in light acclimation in Arabidopsis. Plant Cell 24, 2596-2609. doi: 10.1105/tpc.112.095703

Schneider, A., Steinberger, I., Strissel, H., Kunz, H.-H., Manavski, N., Meurer, J., et al. (2014). The Arabidopsis Tellurite resistance C protein together with ALB3 is involved in Photosystem II protein synthesis. Plant J. 78, 344-356. doi: $10.1111 /$ tpj. 12474

Schuhmann, H., and Adamska, I. (2012). Deg proteases and their role in protein quality control and processing in different subcellular compartments of the plant cell. Physiol. Plant. 145, 224-234. doi: 10.1111/j.1399-3054.2011.01533.x

Schuhmann, H., Mogg, U., and Adamska, I. (2011). A new principle of oligomerization of plant DEG7 protease based on interactions of degenerated protease domains. Biochem. J. 435, 167-174. doi: 10.1042/BJ20101613

Schult, K., Meierhoff, K., Paradies, S., Toller, T., Wolff, P., and Westhoff, P. (2007). The nuclear-encoded factor HCF173 is involved in the initiation of translation of the psbA mRNA in Arabidopsis thaliana. Plant Cell 19, 1329-1346. doi: $10.1105 /$ tpc. 106.042895

Schunemann, D. (2007). Mechanisms of protein import into thylakoids of chloroplasts. Biol. Chem. 388, 907-915. doi: 10.1515/BC.2007.111

Shapiguzov, A., Ingelsson, B., Samol, I., Andres, C., Kessler, F., Rochaix, J. D., et al. (2010). The PPH1 phosphatase is specifically involved in LHCII dephosphorylation and state transitions in Arabidopsis. Proc. Natl. Acad. Sci. U.S.A. 107, 4782-4787. doi: 10.1073/pnas.0913810107

Shen, G., Zhao, J., Reimer, S. K., Antonkine, M. L., Cai, Q., Weiland, S. M., et al. (2002). Assembly of Photosystem I: I. Inactivation of the rubA gene encoding a membrane-associated rubredoxin in the cyanobacterium Synechococcus sp. PCC 7002 causes a loss of Photosystem I activity. J. Biol. Chem. 277, 20343-20354. doi: 10.1074/jbc.M201103200

Shi, L.-X., Hall, M., Funk, C., and Schröder, W. P. (2012). Photosystem II, a growing complex: updates on newly discovered components and low molecular mass proteins. Biochim. Biophys. Acta 1817, 13-25. doi: 10.1016/j.bbabio.2011.08.008

Shimada, H., Mochizuki, M., Ogura, K., Froehlich, J. E., Osteryoung, K. W., Shirano, Y., et al. (2007). Arabidopsis cotyledon-specific chloroplast biogenesis factor CYO1 is a protein disulfide isomerase. Plant Cell 19, 3157-3169. doi: 10.1105/tpc.107.051714

Silva, P., Thompson, E., Bailey, S., Kruse, O., Mullineaux, C. W., Robinson, C., et al. (2003). FtsH is involved in the early stages of repair of Photosystem II in Synechocystis sp. PCC 6803. Plant Cell 15, 2152-2164. doi: 10.1105/tpc.012609

Sirpiö, S., Allahverdiyeva, Y., Suorsa, M., Paakkarinen, V., Vainonen, J., Battchikova, N., et al. (2007). TLP18.3, a novel thylakoid lumen protein regulating Photosystem II repair cycle. Biochem. J. 406, 415-425. doi: 10.1042/BJ20070460

Sirpiö, S., Khrouchtchova, A., Allahverdiyeva, Y., Hansson, M., Fristedt, R., Vener, A. V., et al. (2008). AtCYP38 ensures early biogenesis, correct assembly and sustenance of Photosystem II. Plant J. 55, 639-651. doi: 10.1111/j.1365313X.2008.03532.x

Speiser, A., Haberland, S., Watanabe, M., Wirtz, M., Dietz, K. J., Saito, K., et al. (2015). The significance of cysteine synthesis for acclimation to high light conditions. Front. Plant Sci. 5:776. doi: 10.3389/fpls.2014.00776

Spetea, C., Hundal, T., Lohmann, F., and Andersson, B. (1999). GTP bound to chloroplast thylakoid membranes is required for light-induced, multienzyme degradation of the Photosystem II D1 protein. Proc. Natl. Acad. Sci. U.S.A. 96, 6547-6552. doi: 10.1073/pnas.96.11.6547

Staleva, H., Komenda, J., Shukla, M. K., Slouf, V., Kana, R., Polivka, T., et al. (2015). Mechanism of photoprotection in the cyanobacterial ancestor of plant antenna proteins. Nat. Chem. Biol. 11, 287-291. doi: 10.1038/nchembio. 1755

Suga, M., Akita, F., Hirata, K., Ueno, G., Murakami, H., Nakajima, Y., et al. (2015). Native structure of Photosystem II at $1.95 \AA$ A resolution viewed by femtosecond X-ray pulses. Nature 517, 99-103. doi: 10.1038/nature 13991

Summerfield, T., Winter, R., and Eaton-Rye, J. (2005). Investigation of a requirement for the PsbP-like protein in Synechocystis sp. PCC 6803. Photosynth. Res. 84, 263-268. doi: 10.1007/s11120-004-6431-3

Sun, X., Fu, T., Chen, N., Guo, J., Ma, J., Zou, M., et al. (2010a). The stromal chloroplast Deg7 protease participates in the repair of Photosystem II after photoinhibition in Arabidopsis. Plant Physiol. 152, 1263-1273. doi: 10.1104/pp.109.150722

Sun, X., Ouyang, M., Guo, J., Ma, J., Lu, C., Adam, Z., et al. (2010b). The thylakoid protease Deg1 is involved in Photosystem-II assembly in
Arabidopsis thaliana. Plant J. 62, 240-249. doi: 10.1111/j.1365-313X.2010. 04140.x

Sun, X., Peng, L., Guo, J., Chi, W., Ma, J., Lu, C., et al. (2007). Formation of DEG5 and DEG8 complexes and their involvement in the degradation of photodamaged Photosystem II reaction center D1 protein in Arabidopsis. Plant Cell 19, 1347-1361. doi: 10.1105/tpc.106.049510

Takahashi, K., Takabayashi, A., Tanaka, A., and Tanaka, R. (2014). Functional analysis of light-harvesting-like protein 3 (LIL3) and its light-harvesting chlorophyll-binding motif in Arabidopsis. J. Biol. Chem. 289, 987-999. doi: 10.1074/jbc.M113.525428

Takechi, K., Sodmergen, Murata, M., Motoyoshi, F., and Sakamoto, W. (2000). The YELLOW VARIEGATED (VAR2) locus encodes a homologue of $\mathrm{FtsH}$, an ATP-dependent protease in Arabidopsis. Plant Cell Physiol. 41, 1334-1346. doi: $10.1093 / \mathrm{pcp} / \mathrm{pcd} 067$

Tanaka, R., Rothbart, M., Oka, S., Takabayashi, A., Takahashi, K., Shibata, M., et al. (2010). LIL3, a light-harvesting-like protein, plays an essential role in chlorophyll and tocopherol biosynthesis. Proc. Natl. Acad. Sci. U.S.A. 107, 16721-16725. doi: 10.1073/pnas.1004699107

Tanz, S. K., Kilian, J., Johnsson, C., Apel, K., Small, I., Harter, K., et al. (2012). The SCO2 protein disulphide isomerase is required for thylakoid biogenesis and interacts with LHCB1 chlorophyll a/b binding proteins which affects chlorophyll biosynthesis in Arabidopsis seedlings. Plant J. 69, 743-754. doi: 10.1111/j.1365-313X.2011.04833.x

Thornton, L. E., Ohkawa, H., Roose, J. L., Kashino, Y., Keren, N., and Pakrasi, H. B. (2004). Homologs of plant PsbP and PsbQ proteins are necessary for regulation of Photosystem II activity in the cyanobacterium Synechocystis 6803. Plant Cell 16, 2164-2175. doi: 10.1105/tpc.104.023515

Tikkanen, M., and Aro, E. M. (2012). Thylakoid protein phosphorylation in dynamic regulation of Photosystem II in higher plants. Biochim. Biophys. Acta 1817, 232-238. doi: 10.1016/j.bbabio.2011.05.005

Tikkanen, M., Nurmi, M., Kangasjärvi, S., and Aro, E. M. (2008). Core protein phosphorylation facilitates the repair of photodamaged Photosystem II at high light. Biochim. Biophys. Acta 1777, 1432-1437. doi: 10.1016/j.bbabio.2008.08.004

Torabi, S., Umate, P., Manavski, N., Plöchinger, M., Kleinknecht, L., Bogireddi, H., et al. (2014). PsbN is required for assembly of the Photosystem II reaction center in Nicotiana tabacum. Plant Cell 26, 1183-1199. doi: 10.1105/tpc.113.120444

Umena, Y., Kawakami, K., Shen, J. R., and Kamiya, N. (2011). Crystal structure of oxygen-evolving Photosystem II at a resolution of $1.9 \AA$ A. Nature 473, 55-U65. doi: 10.1038/nature09913

Uniacke, J., and Zerges, W. (2007). Photosystem II assembly and repair are differentially localized in Chlamydomonas. Plant Cell 19, 3640-3654. doi: 10.1105/tpc.107.054882

Urantowka, A., Knorpp, C., Olczak, T., Kolodziejczak, M., and Janska, H. (2005). Plant mitochondria contain at least two $i$-AAA-like complexes. Plant Mol. Biol. 59, 239-252. doi: 10.1007/s11103-005-8766-3

Vasudevan, D., Fu, A., Luan, S., and Swaminathan, K. (2012). Crystal structure of Arabidopsis cyclophilin38 reveals a previously uncharacterized immunophilin fold and a possible autoinhibitory mechanism. Plant Cell 24, 2666-2674. doi: 10.1105/tpc.111.093781

Vener, A. V., Harms, A., Sussman, M. R., and Vierstra, R. D. (2001). Mass spectrometric resolution of reversible protein phosphorylation in photosynthetic membranes of Arabidopsis thaliana. J. Biol. Chem. 276, 6959-6966. doi: 10.1074/jbc.M009394200

Vener, A. V., Rokka, A., Fulgosi, H., Andersson, B., and Herrmann, R. G. (1999). A cyclophilin-regulated PP2A-like protein phosphatase in thylakoid membranes of plant chloroplasts. Biochemistry 38, 14955-14965. doi: 10.1021/bi990971v

Wagner, R., Aigner, H., Pruzinska, A., Jankanpaa, H. J., Jansson, S., and Funk, C. (2011). Fitness analyses of Arabidopsis thaliana mutants depleted of FtsH metalloproteases and characterization of three FtsH6 deletion mutants exposed to high light stress, senescence and chilling. New Phytol. 191, 449-458. doi: 10.1111/j.1469-8137.2011.03684.x

Walter, B., Hristou, A., Nowaczyk, M. M., and Schunemann, D. (2015). In vitro reconstitution of co-translational D1 insertion reveals a role of the cpSec-Alb3 translocase and Vipp1 in Photosystem II biogenesis. Biochem. J. 468, 315-324. doi: 10.1042/BJ20141425

Wang, P., Liu, J., Liu, B., Feng, D., Da, Q., Shu, S., et al. (2013). Evidence for a role of chloroplastic m-type thioredoxins in the biogenesis of Photosystem 
II in Arabidopsis. Plant Physiol. 163, 1710-1728. doi: 10.1104/pp.113. 228353

Wang, Q., Sullivan, R. W., Kight, A., Henry, R. L., Huang, J., Jones, A. M., et al. (2004). Deletion of the chloroplast-localized Thylakoid formation 1 gene product in Arabidopsis leads to deficient thylakoid formation and variegated leaves. Plant Physiol. 136, 3594-3604. doi: 10.1104/pp.104.049841

Wei, L., Guo, J., Ouyang, M., Sun, X., Ma, J., Chi, W., et al. (2010). LPA19, a Psb27 homolog in Arabidopsis thaliana, facilitates D1 protein precursor processing during PSII biogenesis. J. Biol. Chem. 285, 21391-21398. doi: 10.1074/jbc.M110.105064

Westphal, S., Soll, J., and Vothknecht, U. C. (2001). A vesicle transport system inside chloroplasts. FEBS Lett. 506, 257-261. doi: 10.1016/S00145793(01)02931-3

Westphal, S., Soll, J., and Vothknecht, U. C. (2003). Evolution of chloroplast vesicle transport. Plant Cell Physiol. 44, 217-222. doi: 10.1093/pcp/pcg023

Wittenberg, G., Levitan, A., Klein, T., Dangoor, I., Keren, N., and Danon, A. (2014). Knockdown of the Arabidopsis thaliana chloroplast protein disulfide isomerase 6 results in reduced levels of photoinhibition and increased D1 synthesis in high light. Plant J. 78, 1003-1013. doi: 10.1111/tpj.12525

Wu, H. Y., Liu, M. S., Lin, T. P., and Cheng, Y. S. (2011). Structural and functional assays of AtTLP18.3 identify its novel acid phosphatase activity in thylakoid lumen. Plant Physiol. 157, 1015-1025. doi: 10.1104/pp.111.184739

Yamamoto, Y., Inagaki, N., and Satoh, K. (2001). Overexpression and characterization of carboxyl-terminal processing protease for precursor D1 protein: regulation of enzyme-substrate interaction by molecular environments. J. Biol. Chem. 276, 7518-7525. doi: 10.1074/jbc.M008877200

Yamatani, H., Sato, Y., Masuda, Y., Kato, Y., Morita, R., Fukunaga, K., et al. (2013). NYC4, the rice ortholog of Arabidopsis THF1, is involved in the degradation of chlorophyll - protein complexes during leaf senescence. Plant J. 74, 652-662. doi: $10.1111 /$ tpj. 12154

Yin, S., Sun, X., and Zhang, L. (2008). An Arabidopsis ctpA homologue is involved in the repair of Photosystem II under high light. Chin. Sci. Bull. 53, 1021-1026. doi: 10.1007/s11434-008-0153-4

Yu, F., Park, S., and Rodermel, S. R. (2004). The Arabidopsis FtsH metalloprotease gene family: interchangeability of subunits in chloroplast oligomeric complexes. Plant J. 37, 864-876. doi: 10.1111/j.1365-313X.2003.02014.x

Yu, F., Park, S., and Rodermel, S. R. (2005). Functional redundancy of AtFtsH metalloproteases in thylakoid membrane complexes. Plant Physiol. 138, 1957-1966. doi: 10.1104/pp.105 061234

Zaltsman, A., Feder, A., and Adam, Z. (2005a). Developmental and light effects on the accumulation of FtsH protease in Arabidopsis chloroplasts - implications for thylakoid formation and Photosystem II maintenance. Plant J. 42, 609-617. doi: 10.1111/j.1365-313X.2005.02401.x

Zaltsman, A., Ori, N., and Adam, Z. (2005b). Two types of FtsH protease subunits are required for chloroplast biogenesis and Photosystem II repair in Arabidopsis. Plant Cell 17, 2782-2790. doi: 10.1105/tpc.105.035071

Zelisko, A., Garcia-Lorenzo, M., Jackowski, G., Jansson, S., and Funk, C. (2005). AtFtsH6 is involved in the degradation of the light-harvesting complex II during high-light acclimation and senescence. Proc. Natl. Acad. Sci. U.S.A. 102, 13699-13704. doi: 10.1073/pnas.0503472102

Zhang, D., Zhou, G., Liu, B., Kong, Y., Chen, N., Qiu, Q., et al. (2011). HCF243 encodes a chloroplast-localized protein involved in the D1 protein stability of the Arabidopsis Photosystem II complex. Plant Physiol. 157, 608-619. doi: 10.1104/pp.111.183301

Zhang, L., Kato, Y., Otters, S., Vothknecht, U. C., and Sakamoto, W. (2012). Essential role of VIPP1 in chloroplast envelope maintenance in Arabidopsis. Plant Cell 24, 3695-3707. doi: 10.1105/tpc.112.103606

Zhang, L., and Sakamoto, W. (2012). Possible function of VIPP1 in thylakoids: protection but not formation? Plant Signal. Behav. 8:e22860. doi: $10.4161 /$ psb. 22860

Zhang, L., Wei, Q., Wu, W., Cheng, Y., Hu, G., Hu, F., et al. (2009). Activation of the heterotrimeric $\mathrm{G}$ protein alpha-subunit GPAl suppresses the ftsh-mediated inhibition of chloroplast development in Arabidopsis. Plant J. 58, 1041-1053. doi: 10.1111/j.1365-313X.2009.03843.x

Conflict of Interest Statement: The author declares that the research was conducted in the absence of any commercial or financial relationships that could be construed as a potential conflict of interest.

Copyright (c) $2016 \mathrm{Lu}$. This is an open-access article distributed under the terms of the Creative Commons Attribution License (CC BY). The use, distribution or reproduction in other forums is permitted, provided the original author (s) or licensor are credited and that the original publication in this journal is cited, in accordance with accepted academic practice. No use, distribution or reproduction is permitted which does not comply with these terms. 